\title{
A Reduced Complexity Approach to IAA Beamforming for Efficient DOA Estimation of Coherent Sources
}

\author{
Marc Barcelo, Jose Lopez Vicario, and Gonzalo Seco-Granados \\ Group of Signal Processing for Communications and Navigations (SPCOMNAV), Universitat Autonoma de Barcelona, \\ Bellaterra (Cerdanyola del Valles), 08193 Barcelona, Spain
}

Correspondence should be addressed to Jose Lopez Vicario, jose.vicario@uab.es

Received 14 May 2010; Revised 21 September 2010; Accepted 13 December 2010

Academic Editor: M. Pesavento

Copyright (C) 2011 Marc Barcelo et al. This is an open access article distributed under the Creative Commons Attribution License, which permits unrestricted use, distribution, and reproduction in any medium, provided the original work is properly cited.

We address the 2D direction-of-arrival (DOA) estimation problem in scenarios with coherent sources. More specifically, we adopt beamforming solutions based on the iterative adaptive approach (IAA) recently proposed in the literature. The motivation of such adoption mainly comes from the excellent behavior these beamformers provide in scenarios with coherent sources. Nonetheless, these strategies suffer from a prohibitive computational complexity, especially in 2D scenarios. In order to alleviate the, we propose two reduced-complexity (RC) versions of the IAA and IAA based on maximum likelihood (IAA-ML) algorithms. The proposed beamformers are referred to as IAA-RC and IAA-ML-RC and provide similar results to those obtained with their original counterparts. Computational complexity, however, is further reduced. Numerical results presented in the paper show that the computational burden can be decreased by a $52 \%$ with our proposed solutions in the considered scenarios.

\section{Introduction}

Array signal processing is a research area that has been deeply studied in the last decades. Although a wide set of results and advances have been attained, there exist some remaining open problems. Among all of them, the problem of dealing with coherent sources is still a challenge when adaptive beamformers are considered. It is worth noting that this problem is of special interest nowadays. In the context of the Galileo ground mission segment, high-performance tracking stations achieving centimeter level tracking accuracy are required to provide the system with accurate satellite ephemeris and clock prediction models [1]. Tracking stations work in static and controlled scenarios, being the ionospheric perturbations and multipath and interference components the dominant error sources. One of the most promising approaches to cope with multipath and interference signals is the adoption of arrays of antennas at the ground station receivers $[2,3]$. Therefore, the need of deriving beamforming algorithms robust against multipath (coherent) signals is crucial for a successful development of incoming Galileo tracking stations.

In the direction-of-arrival (DOA) estimation problem, in particular, high-resolution results can be attained with the well-known MUSIC [4] and unitary ESPRIT [5] solutions (and their variants). The problem of such subspace-based strategies is that the presence of coherent (or correlated) signals induces a rank deficiency of the source covariance matrix. As a result, the algorithms are not able to properly differentiate the different sources, and performance is seriously degraded. In order to deal with this problem, some preprocessing techniques can be applied to decorrelate the sources such as spatial smoothing $[6,7]$. The problem, however, is that these pre-processing techniques reduce the effective size of the array. As an alternative to the methodologies commented above, one can resort to parametric maximum likelihood (ML) beamforming strategies, [8] since their performance is not degraded in the presence of coherent signals. This is because the parameterization of the received signal takes into account all the sources' 


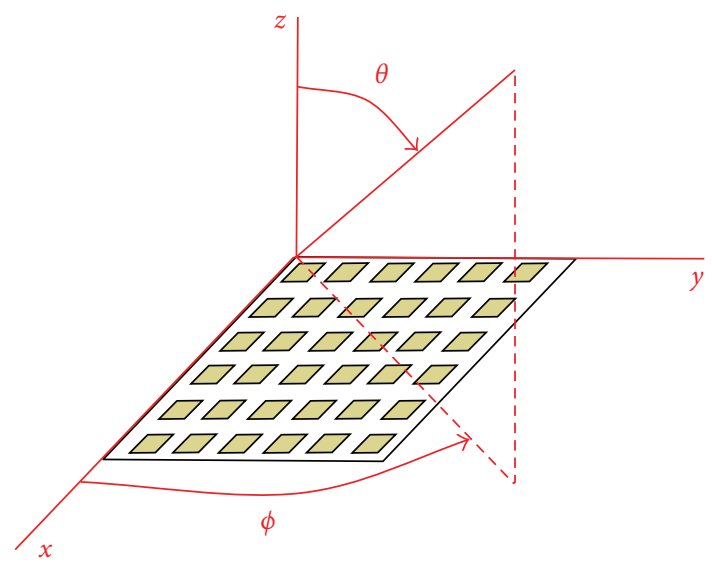

FIgURE 1: Array geometry.

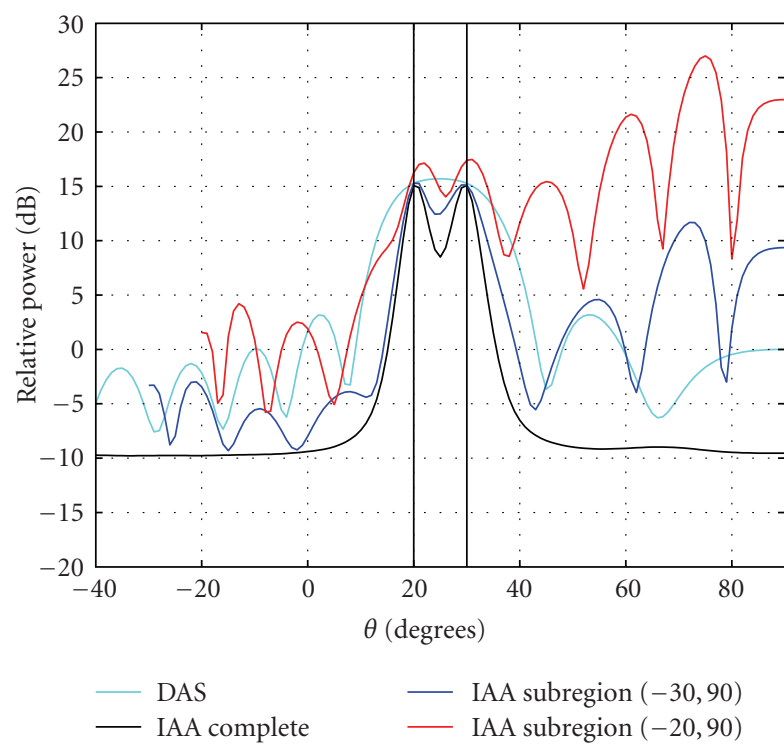

Figure 2: Performance degradation effects of the IAA solution for different degrees of scanning region limitation (linear array, $\theta_{1}=$ $20^{\circ}, \theta_{2}=30^{\circ}, \mathrm{SNR}_{1}=\mathrm{SNR}_{2}=15 \mathrm{~dB}$, and $f_{c}=1575 \mathrm{MHz}$ ).

signals impinging on the array. As an inconvenience, these algorithms require a good knowledge of the scenario and the beamforming derivation results in a complex multidimensional optimization problem, increasing then the computational requirements of the system.

Although a high amount of variants of the strategies mentioned above have been proposed during the last years, the problem of addressing coherent sources in an unknown scenario has not been efficiently solved. Recently, however, a number of variant beamformers based on sparse approaches showing satisfactory results have been proposed [9-11]. These beamformers, referred as iterative adaptive approaches (IAA), have been tested and compared with other robust solutions in scenarios with coherent sources, array perturbations, and finite-sampling effects. As shown in these works, the authors prove that IAA-based algorithms are quite efficient to deal with all these kinds of impairments by providing the most equilibrated strategy in terms of signalto-noise-plus-interference ratio (SNIR) estimation accuracy of DOA, and power of the desired signal. The main drawback of these strategies, nonetheless, is the high computational complexity involved in the beamforming computation, as it is based on the complete scanning of all the visible region of the array.

In this paper, we also consider the adoption of IAA-based beamformers, to address the problem of angle estimation in a 2D environment with coherent sources. Since the computational complexity of IAA is significantly high, specially in $2 \mathrm{D}$ scenarios, we derive two reduced complexity versions of IAA: IAA with reduced complexity (IAA-RC) and IAA based on the maximum likelihood approach with reduced complexity (IAA-ML-RC). As shown in the paper, results obtained with our reduced complexity solutions are quite similar to results provided by the original IAA beamformers but complexity can be significantly reduced.

The rest of the paper is organized as follows. In Section 2, we present the signal model considered in this paper. After that, we review the IAA beamformers in Section 3. In Section 4, we derive the reduced complexity versions of the IAA solutions and analyze the resulting computational complexity. Finally, we analyze the behavior of the proposed algorithms and conclude the paper in Sections 5 and 6, respectively.

\section{Signal Model}

Consider a scenario with $K$ coherent sources and a receiver consisting in an $M$ antennas uniform rectangular array (URA). In particular, these sources are located at angles $\tilde{\boldsymbol{\theta}}=$ $\left[\tilde{\theta}_{1}, \ldots, \tilde{\theta}_{K}\right]$ and $\tilde{\phi}=\left[\tilde{\phi}_{1}, \ldots, \tilde{\phi}_{K}\right]$ where $\pi / 2-\tilde{\theta}_{k}$ and $\tilde{\phi}_{k}$ are the elevation and azimuth (expressed in radians) of the $k$ th source, respectively. By taking into account a linear, nondispersive, and isotropic transmission medium along with the far-field and narrowband assumptions (assumptions attained in a high variety of scenarios related to communication and navigation systems), the received signal vector corresponding to the $n$th snapshot can be modeled as:

$$
\mathbf{x}(n)=\mathbf{A}(\tilde{\boldsymbol{\theta}}, \tilde{\phi}) \mathbf{s}(n)+\mathbf{e}(n),
$$

where $\mathbf{A}(\tilde{\boldsymbol{\theta}}, \tilde{\boldsymbol{\phi}}) \in \mathbb{C}^{M \times K}$ is the matrix gathering the steering vectors associated to the $K$ sources, that is, $\mathbf{A}(\tilde{\boldsymbol{\theta}}, \tilde{\boldsymbol{\phi}})=$ $\left[\mathbf{a}\left(\tilde{\theta}_{1}, \tilde{\phi}_{1}\right), \ldots, \mathbf{a}\left(\tilde{\theta}_{k}, \tilde{\phi}_{k}\right)\right], \mathbf{s}(n) \in \mathbb{C}^{K \times 1}$ is the vector collecting the waveform signals transmitted by the different sources and $\mathbf{e}(n) \in \mathbb{C}^{M \times 1}$ is spatially and temporally white noise.

The beamforming vector is denoted by the vector $\mathbf{w}(n) \in$ $\mathbb{C}^{M \times 1}$ and the beamforming operation can be written as:

$$
y(n)=\mathbf{w}^{H}(n) \mathbf{x}(n) .
$$

Since in this work we consider the use of adaptive beamformers, the covariance matrix must be estimated. In particular, we consider the maximum likelihood estimate 


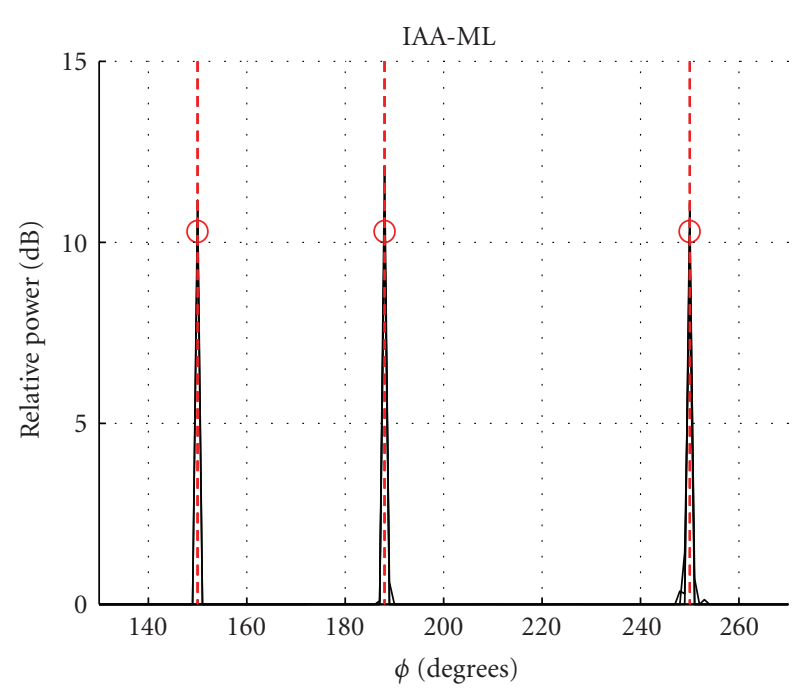

(a)

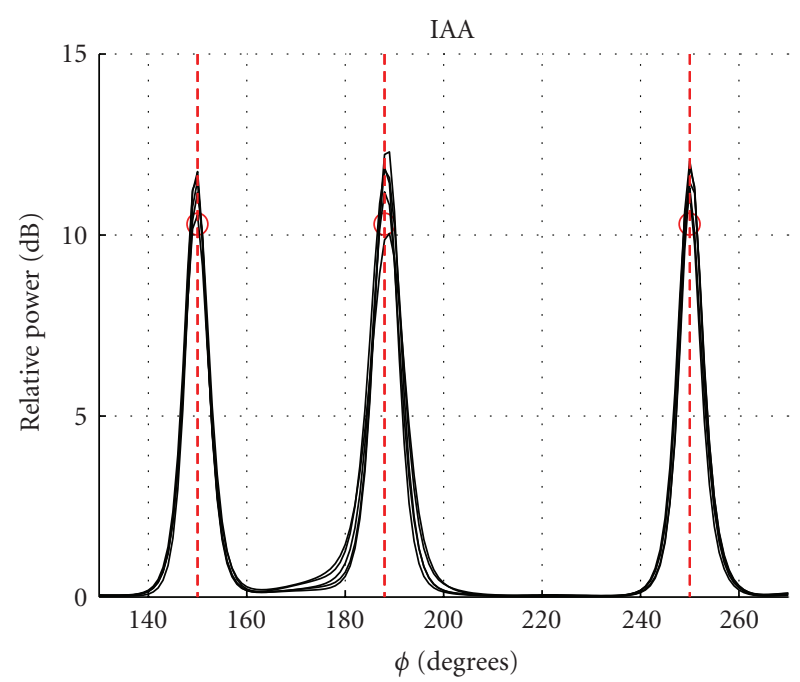

(c)



(b)

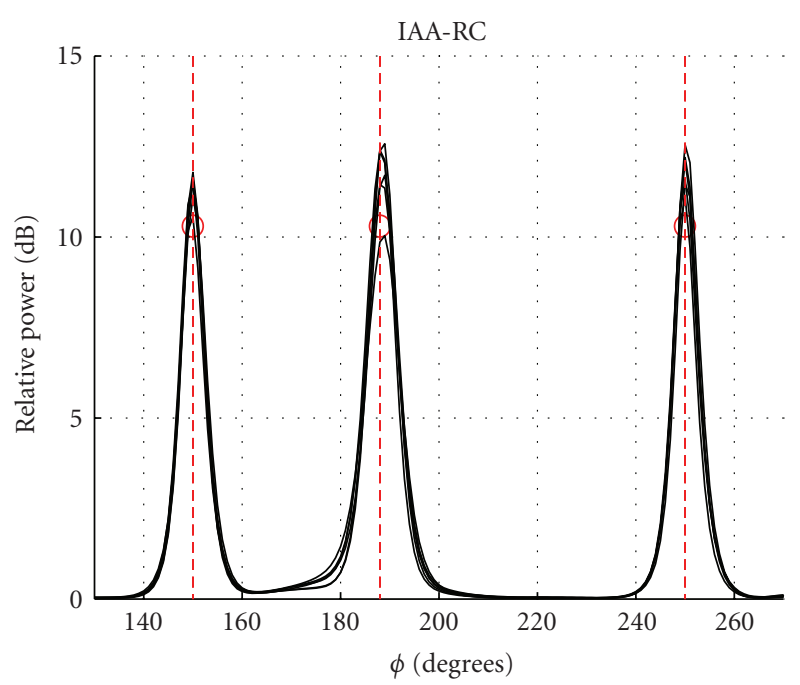

(d)

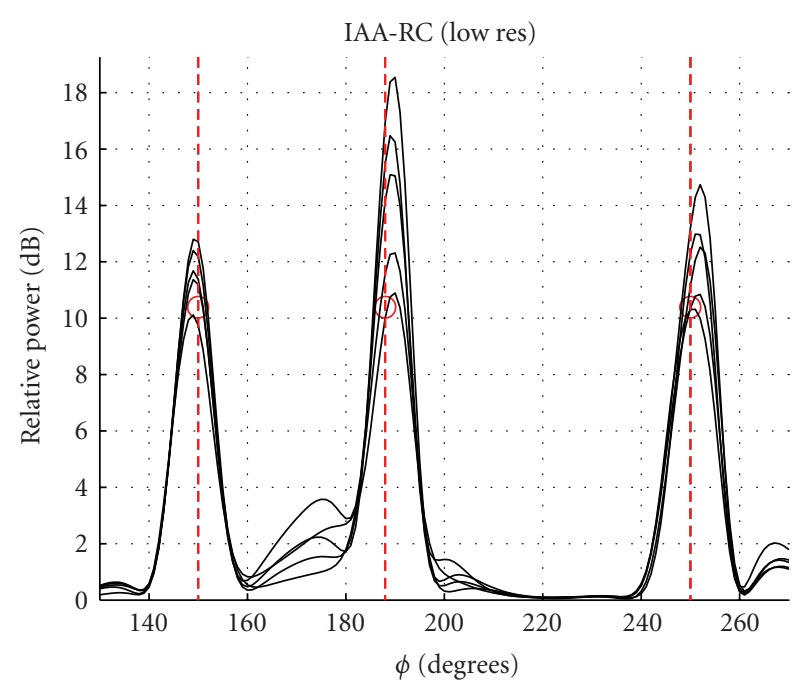

(e)

Figure 3: Spatial estimates of the different beamforming solutions (rectangular array, $\theta_{1}=45^{\circ}, \phi_{1}=150^{\circ}, \theta_{2}=45^{\circ}, \phi_{2}=188^{\circ}, \theta_{3}=45^{\circ}$, $\phi_{3}=250^{\circ}, N=10$ snapshots, $\mathrm{SNR}_{1}=\mathrm{SNR}_{2}=\mathrm{SNR}_{3}=10 \mathrm{~dB}$, noise power equal to $0 \mathrm{~dB}$, and $f_{c}=1575 \mathrm{MHz}$ ). 


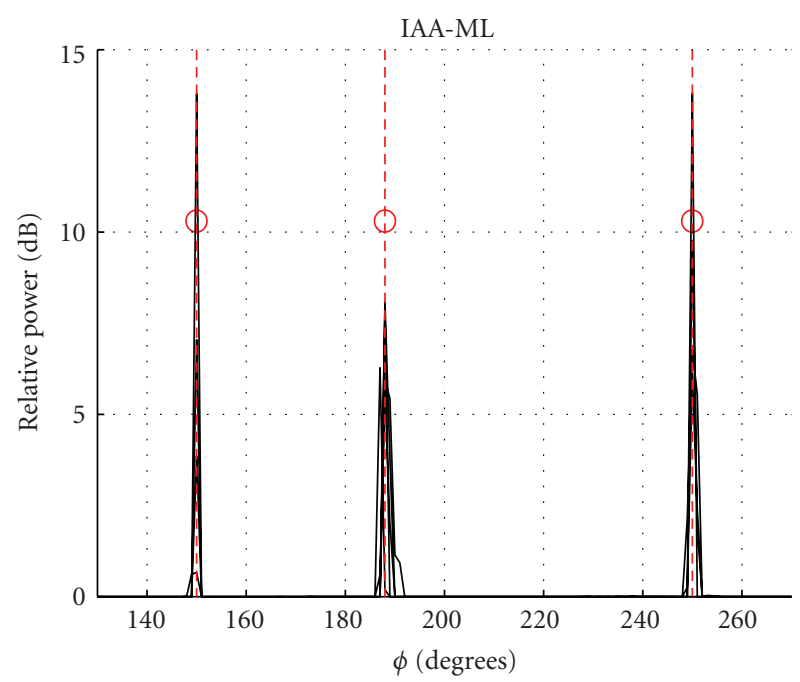

(a)

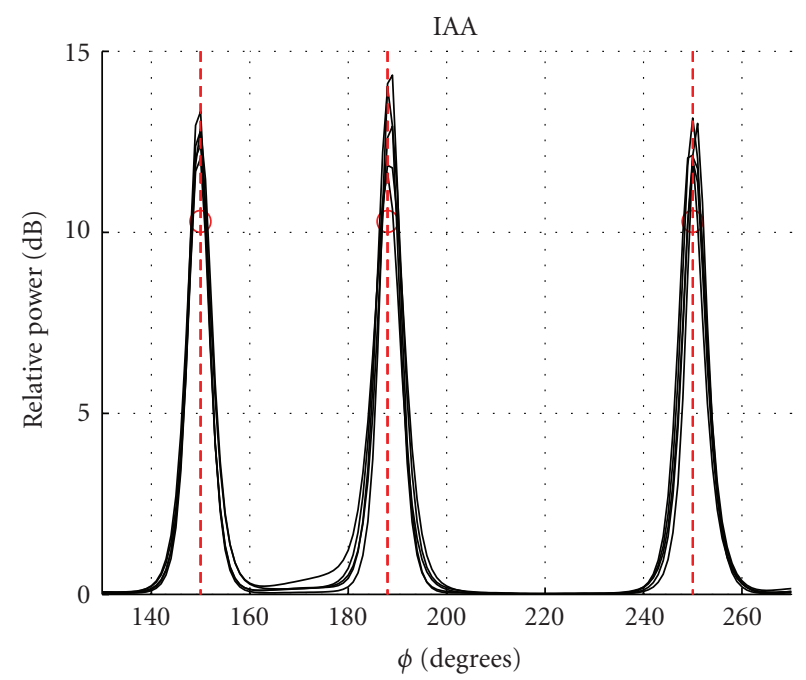

(c)



(b)

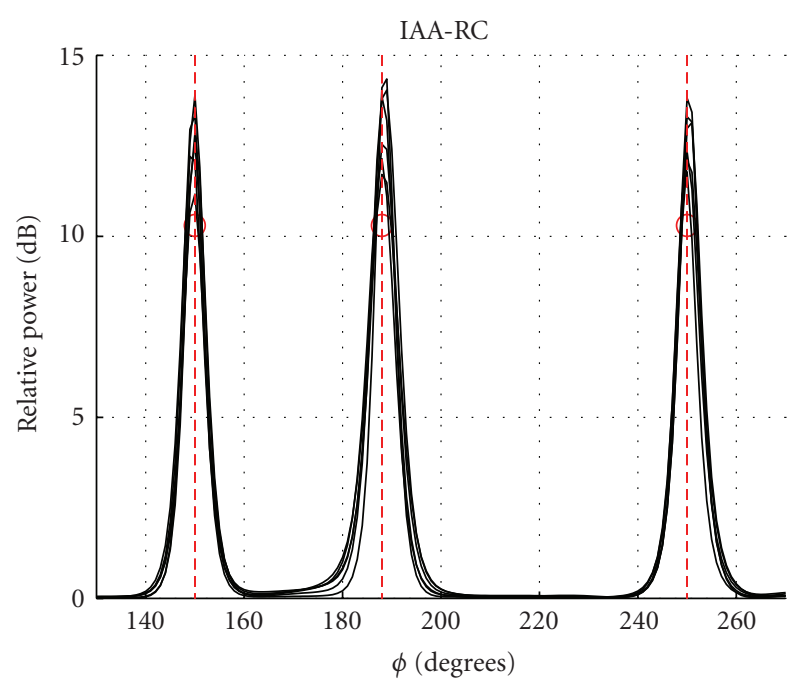

(d)

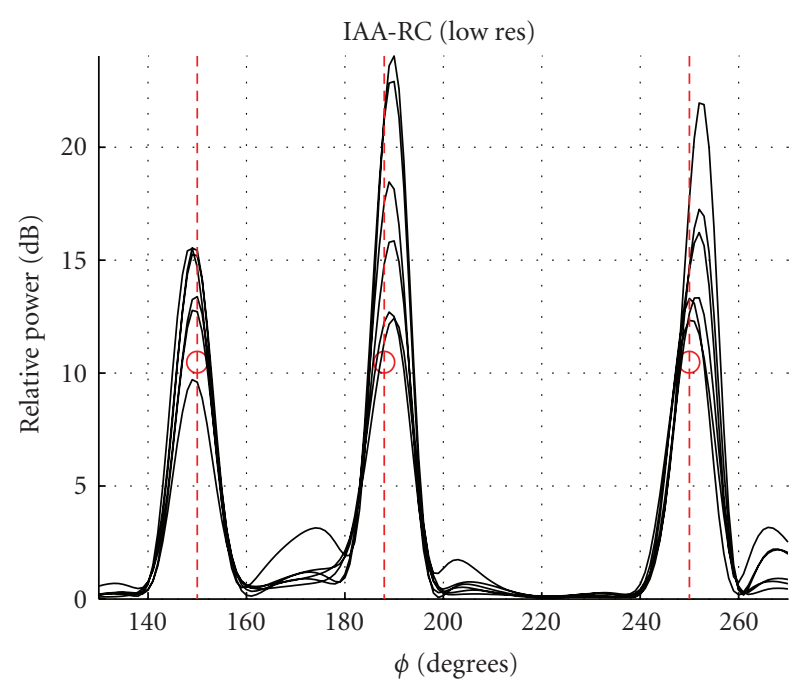

(e)

FigURE 4: Spatial estimates of the different beamforming solutions (rectangular array, $\theta_{1}=45^{\circ}, \phi_{1}=150^{\circ}, \theta_{2}=45^{\circ}, \phi_{2}=188^{\circ}, \theta_{3}=45^{\circ}$, $\phi_{3}=250^{\circ}, N=3$ snapshots, $\mathrm{SNR}_{1}=\mathrm{SNR}_{2}=\mathrm{SNR}_{3}=10 \mathrm{~dB}$, noise power equal to $0 \mathrm{~dB}$, and $\left.f_{c}=1575 \mathrm{MHz}\right)$. 


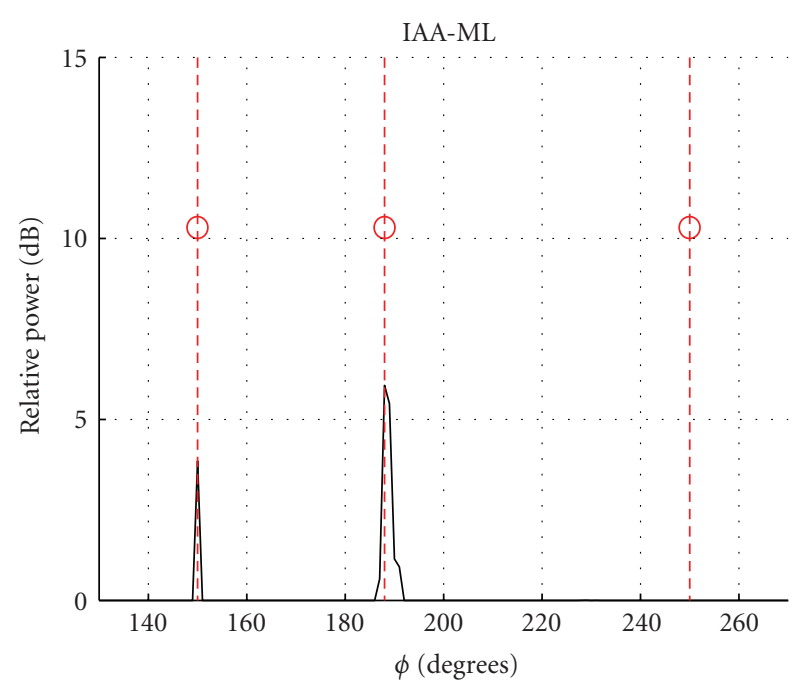

(a)

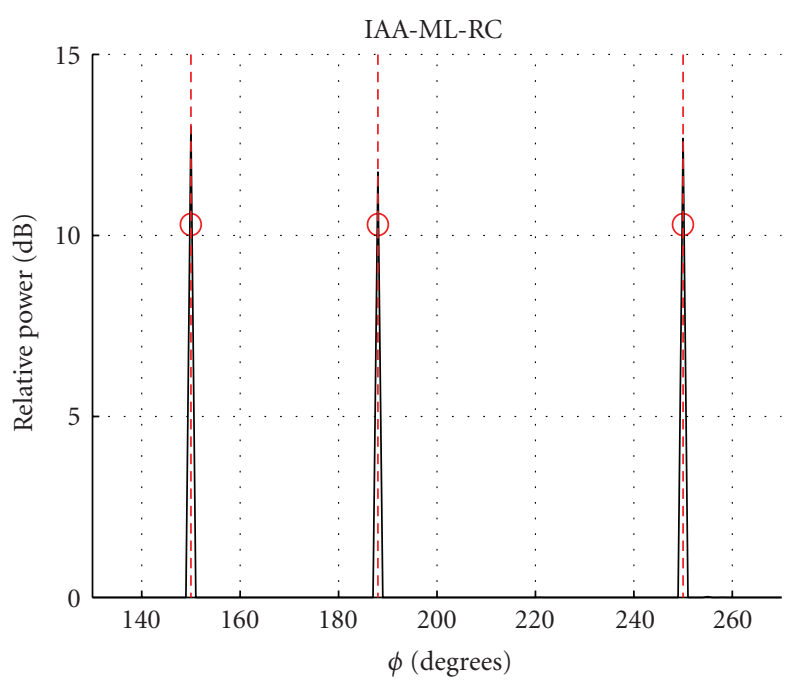

(b)

FIgURE 5: IAA-ML versus IAA-ML-RC comparison (one realization, rectangular array, $\theta_{1}=45^{\circ}, \phi_{1}=150^{\circ}, \theta_{2}=45^{\circ}, \phi_{2}=188^{\circ}, \theta_{3}=45^{\circ}$, $\phi_{3}=250^{\circ}, N=3$ snapshots, $\mathrm{SNR}_{1}=\mathrm{SNR}_{2}=\mathrm{SNR}_{3}=10 \mathrm{~dB}$, noise power equal to $0 \mathrm{~dB}$, and $\left.f_{c}=1575 \mathrm{MHz}\right)$.

of the covariance matrix, which is given by the following expression [12]:

$$
\widehat{\mathbf{R}}_{x}=\frac{1}{N} \sum_{k=0}^{N-1} \mathbf{x}(n-k) \mathbf{x}^{H}(n-k)
$$

with $N$ standing for the number of snapshots considered in the estimation procedure.

Concerning the adoption of a rectangular array, the motivation of such geometry option comes from the fact that this is a practical and feasible solution for practical implementation (reproducibility of antenna conditions, ease of calibration and manufacturing, etc.). It is worth noting, however, that the proposed beamforming solution is independent of the selected geometry, and URA has been considered as it is a common choice adopted by many applications and systems for radar, sonar, and communications. In this work in particular, we consider a $\sqrt{M} \times \sqrt{M}$ antenna deployment where antenna separation is equal to $d=\lambda / 2$, being $\lambda=c / f_{c}$ the wavelength of the incoming signals and $f_{c}$ standing for the carrier frequency. By taking into account the proposed deployment (see Figure 1), the steering vector for each of the sources can be written as [12]

$$
\mathbf{a}\left(\tilde{\theta}_{k}, \tilde{\phi}_{k}\right)=\left[\begin{array}{c}
1 \\
e^{-j(2 \pi d / \lambda) \cos \tilde{\theta}_{k} \cos \tilde{\phi}_{k}} \\
\cdots \\
e^{-j(2 \pi d / \lambda)\left(\cos \tilde{\theta}_{k} \cos \tilde{\phi}_{k}+\cos \tilde{\theta}_{k} \sin \tilde{\phi}_{k}\right)} \\
\cdots \\
e^{-j(2 \pi d / \lambda)(\sqrt{M}-1)\left(\cos \tilde{\theta}_{k} \cos \tilde{\phi}_{k}+\cos \tilde{\theta}_{k} \sin \tilde{\phi}_{k}\right)}
\end{array}\right] .
$$

\section{Review of IAA Beamforming}

The iterative adaptive approach (IAA) beamforming has been recently studied in several works presented in the context of array signal processing $[9,10]$ and multiple-input multiple-output (MIMO) radar [11]. Such beamformer falls into the category of sparse approaches (i.e., a higher number of sources than the actual one is considered) and results from a weighted least-squares problem, where the covariance matrix of the incoming signals is iteratively computed. IAA shows an excellent behavior especially in scenarios with coherent sources. More specifically, the authors in [9] compared the IAA beamformer with other robust approaches and showed that this option is the most equilibrated strategy in terms of SNIR, estimation accuracy of DOA, and power of the desired signal. In that paper, it is also considered the ML version of IAA which provides improved resolution in terms of DOA estimation.

In this work, we adopt both the IAA and IAA-ML solutions for 2D DOA estimation. Further details of the algorithms can be found in the references above, but in this section, we provide a brief review of the two considered approaches.

3.1. IAA. The IAA solution consists of defining a scanning grid of $L$ directions by constructing a set of $L$ steering vectors $\mathbf{A}(\boldsymbol{\theta}, \boldsymbol{\phi})=\left[\mathbf{a}\left(\theta_{1}, \phi_{1}\right), \ldots, \mathbf{a}\left(\theta_{L}, \phi_{L}\right)\right]$, where $\boldsymbol{\theta}=\left[\theta_{1}, \ldots, \theta_{L}\right]$ and $\phi=\left[\phi_{1}, \ldots, \phi_{L}\right]$ are the vectors containing the angles considered at the scanning grid. In a $2 \mathrm{D}$ angle estimation problem, the scanning matrix $\mathbf{A}(\boldsymbol{\theta}, \boldsymbol{\phi})$ must cover the region $\left(0^{\circ} \leq \theta \leq 90^{\circ}, 0^{\circ} \leq \phi \leq 360^{\circ}\right)$. By considering one degree of resolution, for instance, this fact implies that this matrix should contain $L=360 \times 90$ steering vectors related to the all possible directions in the grid. Once this scanning grid is defined, the algorithm estimates the powers at each direction and gathers them in matrix $\hat{\mathbf{P}}=\operatorname{diag}\left\{\hat{P}_{1}, \ldots, \hat{P}_{L}\right\}$. After that, the beamformer for each direction $l$ is computed as

$$
\mathbf{w}_{l}^{H}=\frac{\mathbf{a}^{H}\left(\theta_{l}, \phi_{l}\right) \overline{\mathbf{R}}^{-1}}{\mathbf{a}^{H}\left(\theta_{l}, \phi_{l}\right) \overline{\mathbf{R}}^{-1} \mathbf{a}\left(\theta_{l}, \phi_{l}\right)},
$$




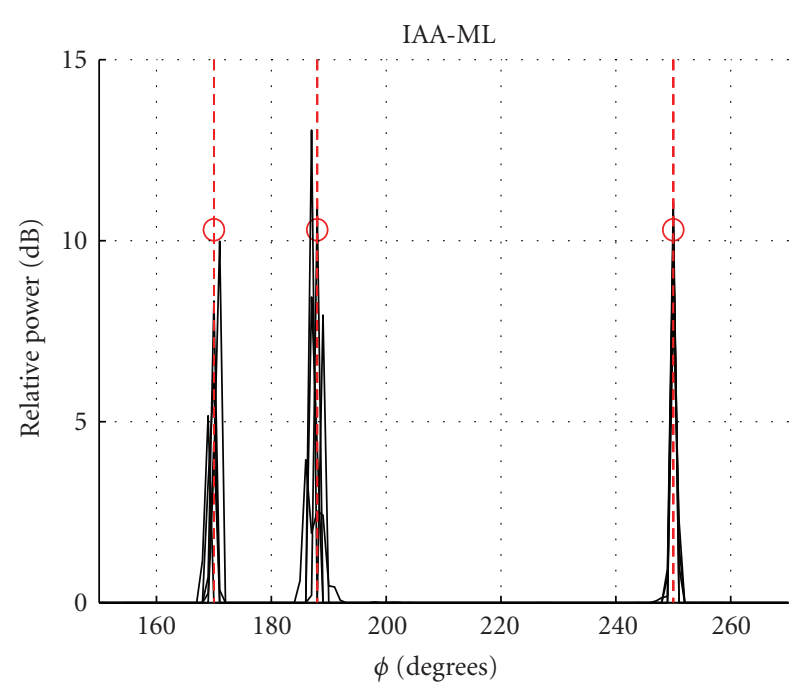

(a)

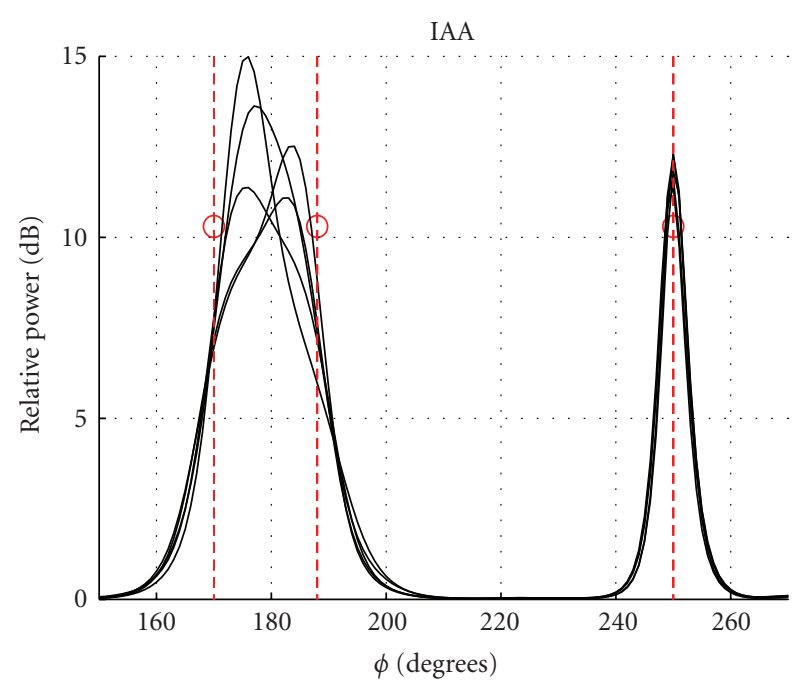

(c)

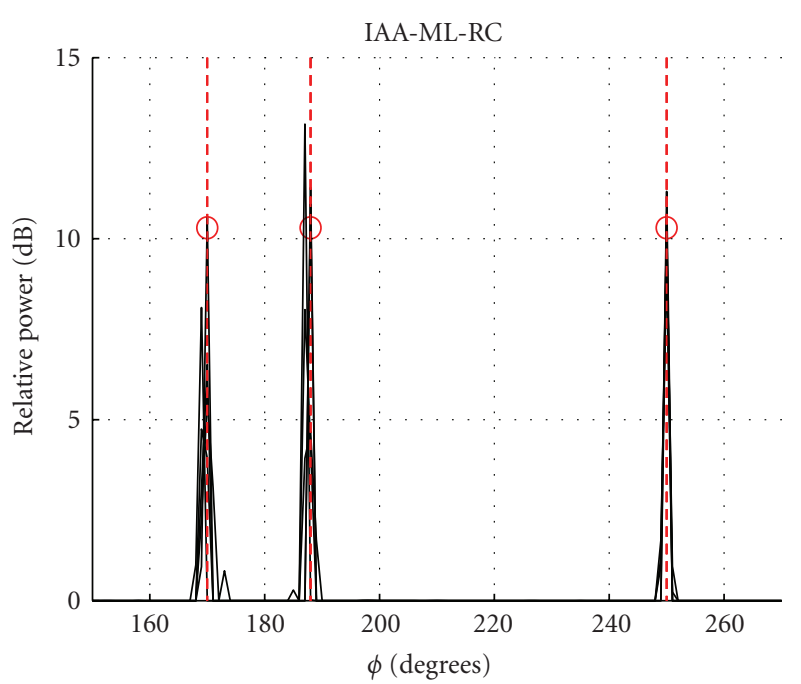

(b)

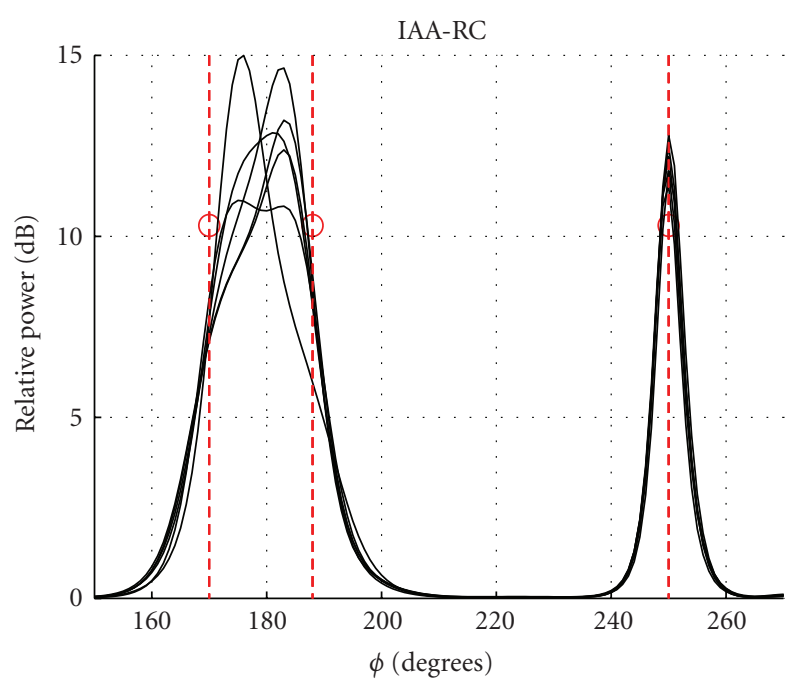

(d)

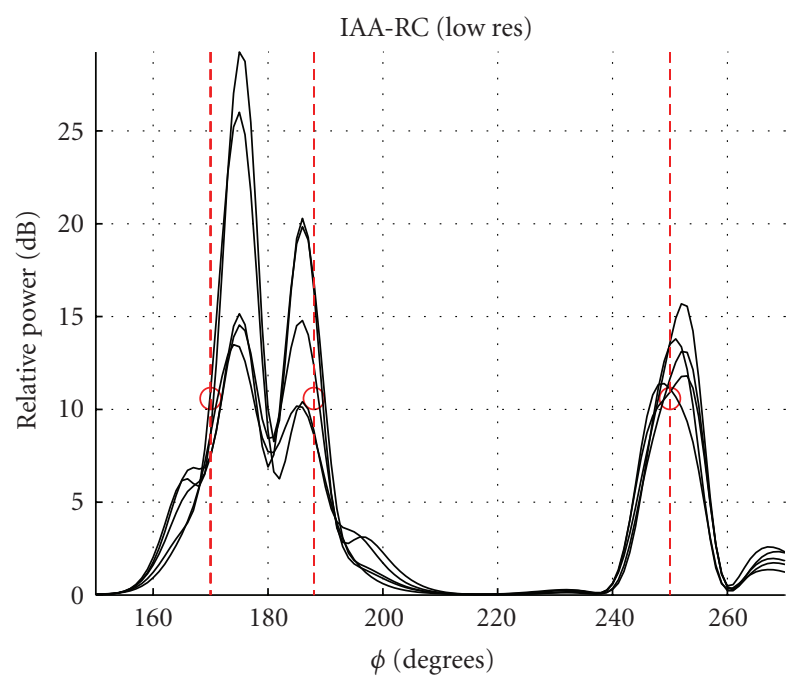

(e)

FIGURE 6: Spatial estimates of the different beamforming solutions (rectangular array, $\theta_{1}=45^{\circ}, \phi_{1}=170^{\circ}, \theta_{2}=45^{\circ}, \phi_{2}=188^{\circ}, \theta_{3}=45^{\circ}$, $\phi_{3}=250^{\circ}, N=10$ snapshots, $\mathrm{SNR}_{1}=\mathrm{SNR}_{2}=\mathrm{SNR}_{3}=10 \mathrm{~dB}$, noise power equal to $0 \mathrm{~dB}$, and $f_{c}=1575 \mathrm{MHz}$ ). 


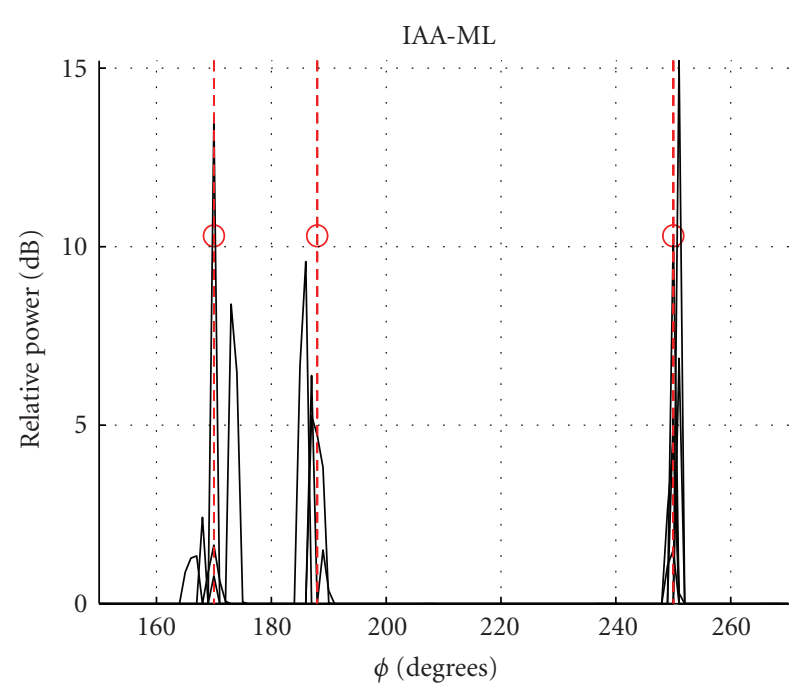

(a)

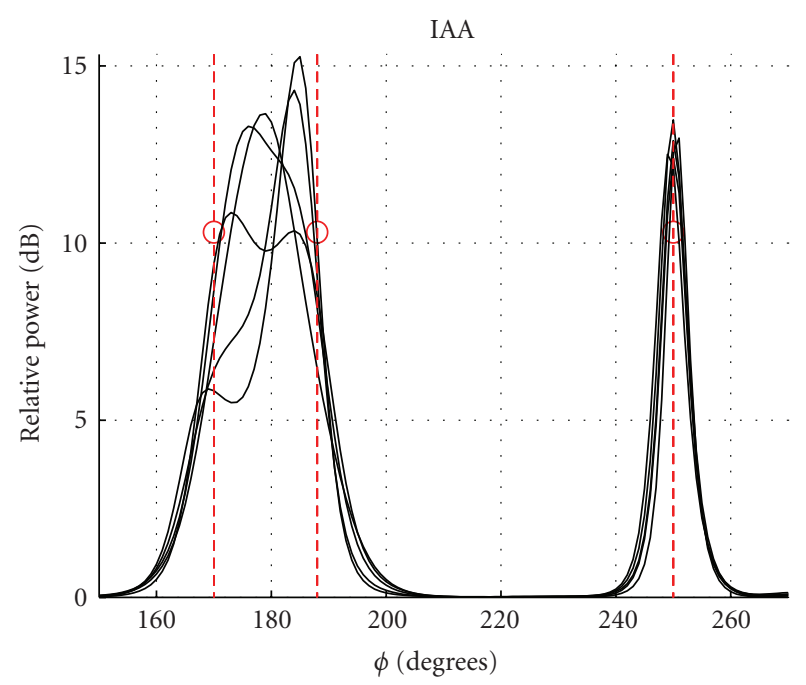

(c)



(b)

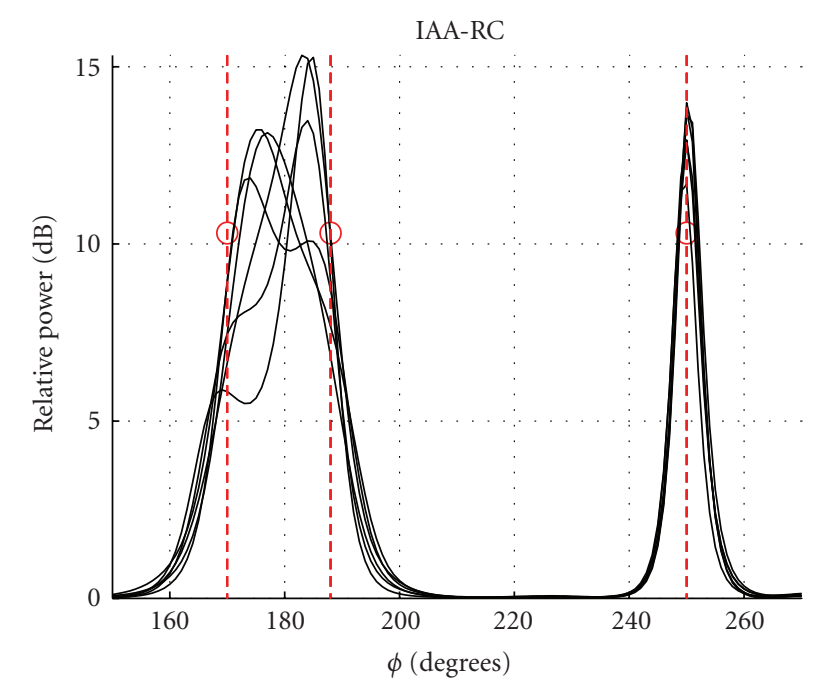

(d)

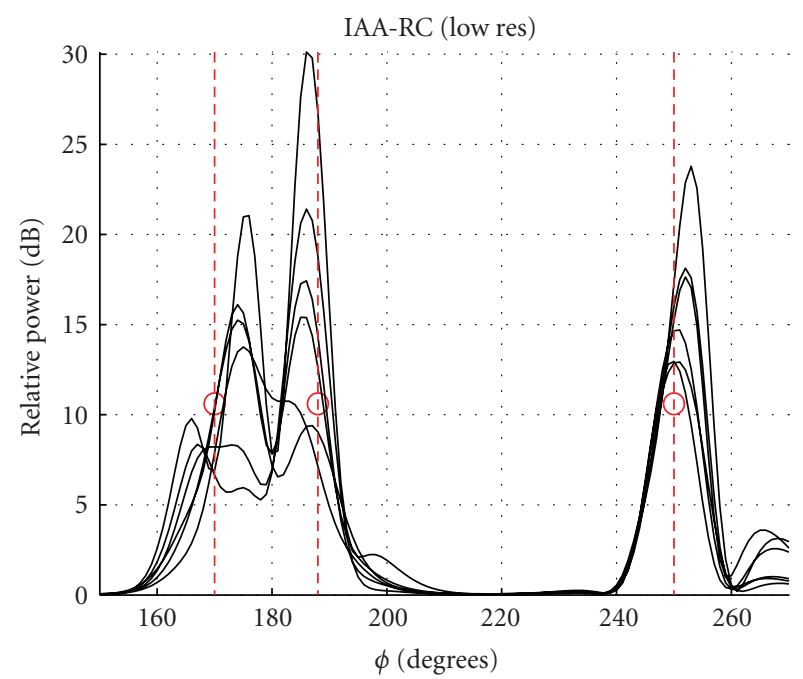

(e)

FigURE 7: Spatial estimates of the different beamforming solutions (rectangular array, $\theta_{1}=45^{\circ}, \phi_{1}=170^{\circ}, \theta_{2}=45^{\circ}, \phi_{2}=188^{\circ}, \theta_{3}=45^{\circ}$, $\phi_{3}=250^{\circ}, N=3$ snapshots, $\mathrm{SNR}_{1}=\mathrm{SNR}_{2}=\mathrm{SNR}_{3}=10 \mathrm{~dB}$, noise power equal to $0 \mathrm{~dB}$, and $f_{c}=1575 \mathrm{MHz}$ ). 


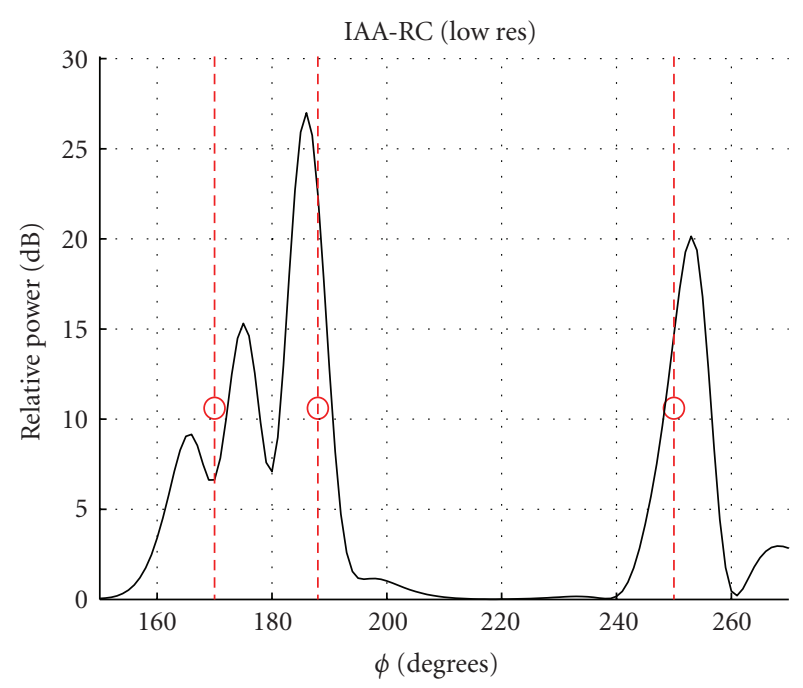

FIGURE 8: IAA-RC (low-res) spatial estimate (one realization, rectangular array, $\theta_{1}=45^{\circ}, \phi_{1}=170^{\circ}, \theta_{2}=45^{\circ}, \phi_{2}=188^{\circ}, \theta_{3}=$ $45^{\circ}, \phi_{3}=250^{\circ}, N=3$ snapshots, $\mathrm{SNR}_{1}=\mathrm{SNR}_{2}=\mathrm{SNR}_{3}=10 \mathrm{~dB}$, noise power equal to $0 \mathrm{~dB}$, and $f_{c}=1575 \mathrm{MHz}$ ).

$$
\begin{aligned}
& \mathbf{A}(\boldsymbol{\theta}, \boldsymbol{\phi})=\left[\mathbf{a}\left(\theta_{1}, \phi_{1}\right), \ldots, \mathbf{a}\left(\theta_{L}, \phi_{L}\right)\right] \\
& \widehat{s}_{l}(n)=\mathbf{a}^{H}\left(\theta_{l}, \phi_{l}\right) \mathbf{x}(n) / M \quad n=1, \ldots, N ; l=1, \ldots, L \\
& \widehat{P}_{l}=(1 / N) \sum_{n=1}^{N}\left|\widehat{s}_{l}(n)\right|^{2} \quad l=1, \ldots, L \\
& \widehat{\mathbf{P}}=\operatorname{diag}\left\{\widehat{P}_{1}, \ldots, \hat{P}_{L}\right\} \\
& \hat{\mathbf{R}}_{x}=(1 / N) \sum_{k=0}^{N-1} \mathbf{x}(n-k) \mathbf{x}^{H}(n-k) \\
& \text { repeat } \\
& \quad \overline{\mathbf{R}}=\mathbf{A}(\boldsymbol{\theta}, \boldsymbol{\phi}) \widehat{\mathbf{P}}^{H}(\boldsymbol{\theta}, \boldsymbol{\phi}) \\
& \quad \text { for } l=1, \ldots, L \\
& \quad \mathbf{w}_{l}^{H}=\mathbf{a}^{H}\left(\theta_{l}, \phi_{l}\right) \overline{\mathbf{R}}^{-1} / \mathbf{a}^{H}\left(\theta_{l}, \phi_{l}\right) \overline{\mathbf{R}}^{-1} \mathbf{a}\left(\theta_{l}, \phi_{l}\right) \\
& \quad \hat{P}_{l}=\mathbf{w}_{l}^{H} \hat{\mathbf{R}}_{x} \mathbf{w}_{l} \\
& \quad \text { end } \\
& \text { Until convergence }
\end{aligned}
$$

Algorithm 1: IAA algorithm [9].

where $\overline{\mathbf{R}}$ is an estimate of the covariance matrix iteratively computed by considering the received signal $\mathbf{x}(n)$ for $N$ snapshots as presented in Algorithm 1.

Notice that the beamforming solution is similar to the classical Capon approach, and the robustness against array perturbations and coherent signals comes from two facts: (1) matrix $\hat{\mathbf{P}}$ is defined by considering that the sources are uncorrelated, and (2) the covariance matrix is estimated by taking into account the power arriving from the directions where the different beamformers are pointing. Note that power estimates are computed by taking into account the sample covariance matrix $\hat{\mathbf{R}}_{x}$. Finally, it is worth noting that 12 iterations are usually required for algorithm convergence. The convergence criterion is based on the evolution of the estimated powers. By defining $\hat{\mathbf{p}}^{s}$ as the vector containing the power estimates at the sth iteration of the algorithm, the convergence is considered to be achieved when $\| \hat{\mathbf{p}}^{s}-$ $\hat{\mathbf{p}}^{s-1}\|/\| \hat{\mathbf{p}}^{s-1} \|$ saturates for values lower than $10^{-3}$. For the rest of algorithms presented in the paper, the same convergence criterion is applied.

3.2. IAA-ML. In this case, sources' powers in matrix $\hat{\mathbf{P}}$ are obtained by resorting to the ML criterion. By assuming that the received signal vector $\mathbf{x}(n)$ is a complex multivariate Gaussian vector, this is done by minimizing the negative log-likelihood function of $\mathbf{x}(n)$. As shown in $[9,10]$, an iterative procedure is again required, which is summarized in Algorithm 2.

Once the convergence is attained and matrix $\hat{\mathbf{P}}$ is estimated, the beamforming vector is finally computed. To do so, the MMSE estimate of the signal waveforms $\mathbf{s}(n), n=$ $1, \ldots, N$, given the observations $\mathbf{x}(n), n=1, \ldots, N$, is employed

$$
\widehat{\mathbf{s}}(n)=\mathbf{W}^{H} \mathbf{x}(n)=\widehat{\mathbf{P}} \mathbf{A}^{H}(\boldsymbol{\theta}, \boldsymbol{\phi})\left(\mathbf{A}(\boldsymbol{\theta}, \boldsymbol{\phi}) \hat{\mathbf{P}} \mathbf{A}^{H}(\boldsymbol{\theta}, \boldsymbol{\phi})\right)^{-1} \mathbf{x}(n) .
$$

More specifically, the IAA-ML beamformer for the $l$ th source is obtained by considering the $l$ th column of matrix $\mathbf{W}$ in the equation above, that is:

$$
\mathbf{w}_{l}=[\mathbf{W}]_{l} .
$$

It is worth noting, however, that since this paper is focused on the use of IAA-ML for angle of arrival estimation, the computation of the beamforming solution is not needed. This is because the angle is estimated by observing the power values at the different directions. In the IAA case, nonetheless, the beamforming computation is required as the power estimates at each iteration are updated with an expression depending on the beamforming weights. In this case, algorithm convergence can also be attained with 12 iterations.

\section{Proposed Reduced Complexity Beamformers}

The main problem of the IAA-based beamformers is the need of scanning the whole visible region. As mentioned above, $L=360 \times 90$ directions should be taken into account in the beamforming computation in the case that one degree of resolution is considered ( $L=32400$ beamformers must be computed at each algorithm iteration!).

Although one can restrict the problem to a smaller region (assuming that some information about the sources' positions is available), one must compute the powers for the entire space. This is because the beamforming solution is based on the powers that are estimated at each direction of the scanning matrix. If a source is not present in a given position, the estimated power there is related to the noise term, and this information is also valuable for the beamforming computation. See for instance Figure 2, where we consider a 1D angle estimation problem with a linear array and two sources (located at $\theta_{1}=20^{\circ}$ and $\theta_{2}=30^{\circ}$ ). For the sake of comparison, we also include the conventional delay-andsum (DAS) beamformer. As observed, the IAA solution is able to separate both sources, and angle estimation accuracy 


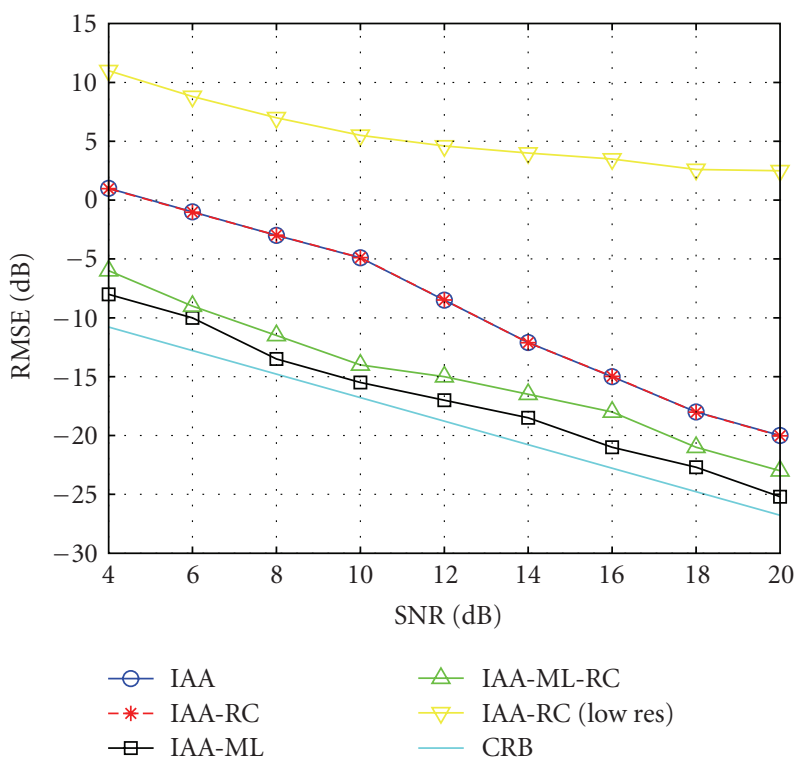

(a)

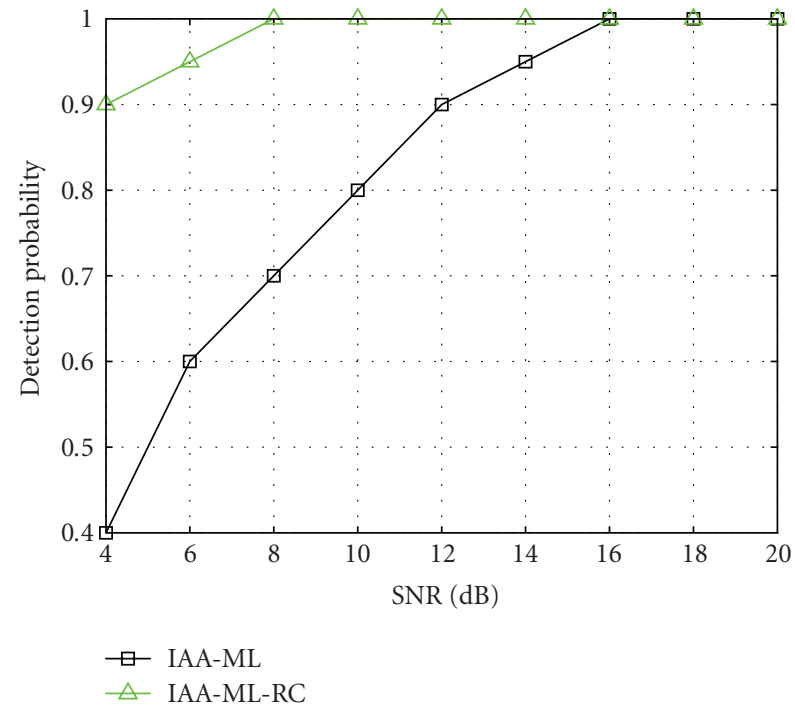

(b)

FIGURE 9: (a) RMSE of the different beamforming solutions. (b) IAA-ML versus IAA-ML-RC detection probability (rectangular array, $\theta_{1}=$ $45^{\circ}, \phi_{1}=150^{\circ}, \theta_{2}=45^{\circ}, \phi_{2}=188^{\circ}, \theta_{3}=45^{\circ}, \phi_{3}=250^{\circ}, N=10$ snapshots, noise power equal to $0 \mathrm{~dB}$, and $f_{c}=1575 \mathrm{MHz}$ ).

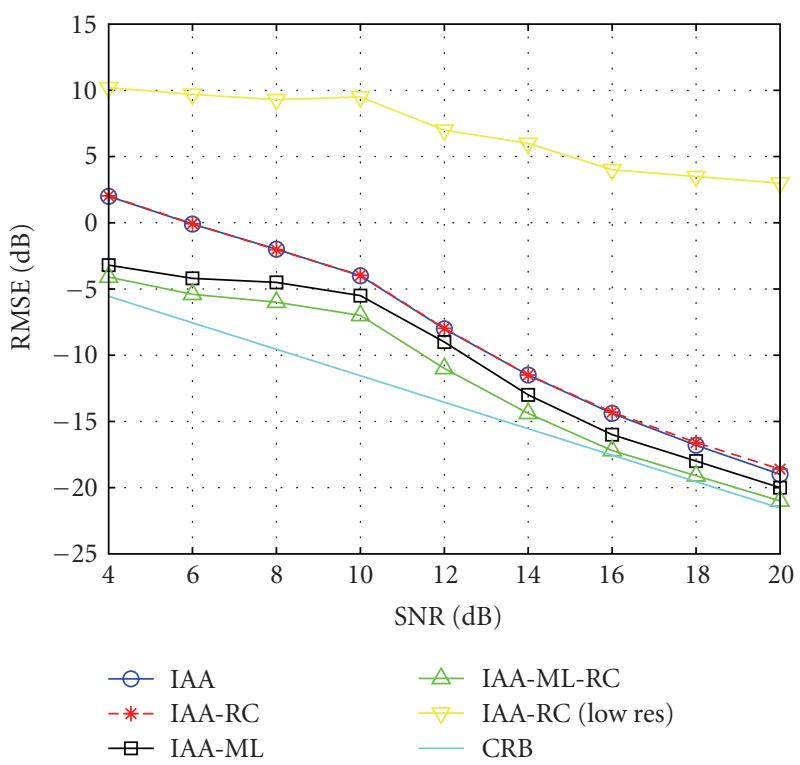

(a)

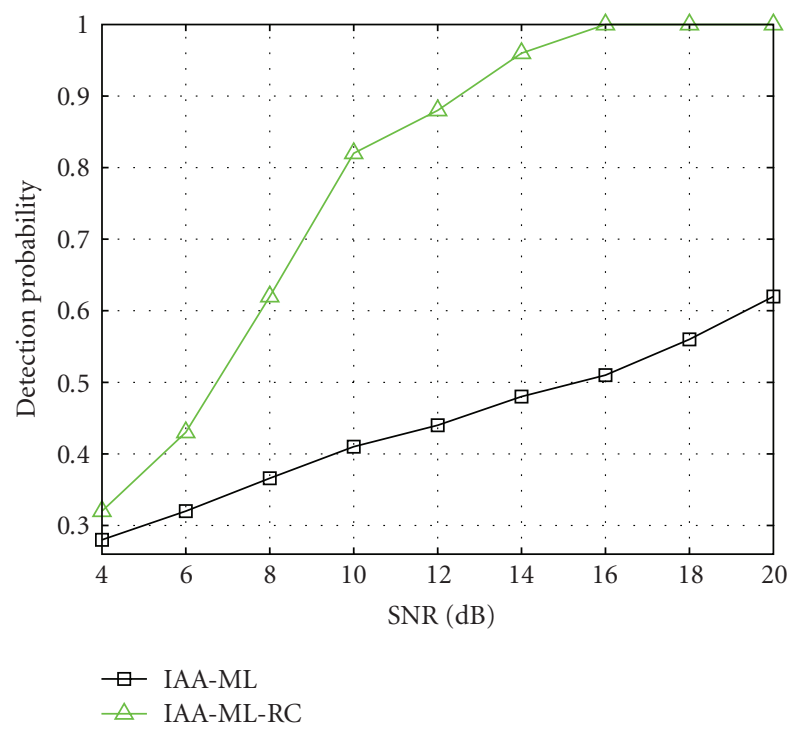

(b)

FIGURE 10: (a) RMSE of the different beamforming solutions. (b) IAA-ML versus IAA-ML-RC detection probability (rectangular array, $\theta_{1}=45^{\circ}, \phi_{1}=150^{\circ}, \theta_{2}=45^{\circ}, \phi_{2}=188^{\circ}, \theta_{3}=45^{\circ}, \phi_{3}=250^{\circ}, N=3$ snapshots, noise power equal to $0 \mathrm{~dB}$, and $f_{c}=1575 \mathrm{MHz}$ ).

is quite better than that provided by the DAS solution. In this case, matrix $\mathbf{A}(\boldsymbol{\theta}, \phi)$ covers the range $-90^{\circ} \leq \theta \leq 90^{\circ}$ (recall that here, we consider a linear array) with one degree of resolution. By restricting the matrix to the region $-30^{\circ} \leq$ $\theta \leq 90^{\circ}$ performance is degraded, obtaining a dramatic performance loss when that region $-20^{\circ} \leq \theta \leq 90^{\circ}$ is considered. This is because the beamformer does not take care of the omitted regions, and high levels of noise are introduced.

The main objective of this paper is to propose reducedcomplexity versions of the IAA beamforming solutions presented in the previous section. To do so, we focus on the 


$$
\begin{aligned}
& \mathbf{A}(\boldsymbol{\theta}, \boldsymbol{\phi})=\left[\mathbf{a}\left(\theta_{1}, \phi_{1}\right), \ldots, \mathbf{a}\left(\theta_{L}, \phi_{L}\right)\right] \\
& \hat{s}_{l}(n)=\mathbf{a}^{H}\left(\theta_{l}, \phi_{l}\right) \mathbf{x}(n) / M \quad n=1, \ldots, N ; l=1, \ldots, L \\
& \widehat{P}_{l}=(1 / N) \sum_{n=1}^{N}\left|\hat{s}_{l}(n)\right|^{2} \quad l=1, \ldots, L \\
& \hat{\mathbf{P}}=\operatorname{diag}\left\{\hat{P}_{1}, \ldots, \hat{P}_{L}\right\} \\
& \overline{\mathbf{R}}^{-1}=\left(\mathbf{A}(\boldsymbol{\theta}, \boldsymbol{\phi}) \hat{\mathbf{P}} \mathbf{A}^{H}(\boldsymbol{\theta}, \boldsymbol{\phi})\right)^{-1} \\
& \hat{\mathbf{R}}_{x}=(1 / N) \sum_{k=0}^{N-1} \mathbf{x}(n-k) \mathbf{x}^{H}(n-k) \\
& \text { Repeat } \\
& \text { Sort } i_{1}, \ldots, i_{L} \text { such that } \hat{P}_{i_{1}} \leq \hat{P}_{i_{2}} \leq \cdots \leq \hat{P}_{i_{L}} \\
& \text { for } l=1, \ldots, L \\
& \hat{P}_{i_{l}}^{\text {prev }}=\hat{P}_{i_{l}} \\
& \widehat{P}_{i_{l}}=\max \left(0, \hat{P}_{i_{l}}+\mathbf{a}^{H}\left(\theta_{i_{l}}, \phi_{i_{l}}\right) \overline{\mathbf{R}}^{-1}\left(\hat{\mathbf{R}}_{x}-\overline{\mathbf{R}}\right) \overline{\mathbf{R}}^{-1} \mathbf{a}\left(\theta_{i_{l}}, \phi_{i_{l}}\right) /\left(\mathbf{a}^{H}\left(\theta_{i_{l}}, \phi_{i_{l}}\right) \overline{\mathbf{R}}^{-1} \mathbf{a}\left(\theta_{i_{l}}, \phi_{i_{l}}\right)\right)^{2}\right) \\
& \overline{\mathbf{R}}^{-1}=\overline{\mathbf{R}}^{-1}-\left(\hat{P}_{i_{l}}-\hat{P}_{i_{l}}^{\text {prev }}\right) \overline{\mathbf{R}}^{-1} \mathbf{a}\left(\theta_{i_{l}}, \phi_{i_{l}}\right) \mathbf{a}^{H}\left(\theta_{i_{l}}, \phi_{i_{l}}\right) \overline{\mathbf{R}}^{-1} / 1+\left(\hat{P}_{i_{l}}-\hat{P}_{i_{l}}^{\text {prev }}\right) \mathbf{a}^{H}\left(\theta_{i_{l}}, \phi_{i_{l}}\right) \overline{\mathbf{R}}^{-1} \mathbf{a}\left(\theta_{i_{l}}, \phi_{i_{l}}\right)
\end{aligned}
$$

Until convergence

Algorithm 2: IAA-ML algorithm [9].

problem presented above and carefully address the power updating at the different directions of matrix $\mathbf{A}(\boldsymbol{\theta}, \boldsymbol{\phi})$ with the aim of reducing the number of required operations. As a result, we derive two reduced-complexity beamforming solutions as presented below.

4.1. Solution 1: IAA-RC. First, we propose a reduced complexity version of the IAA beamformer named as IAARC. As mentioned above, the main drawback of IAAbased schemes is the need of considering the whole visible region in the scanning matrix $\mathbf{A}(\boldsymbol{\theta}, \boldsymbol{\phi})$. Our experiments, however, revealed that the main problem arises in the lack of knowledge of the powers at the omitted directions. In other words, the reason why reducing the scanning space implies a dramatic performance degradation is due to the fact we are considering null powers in such directions. Indeed, this problem can be alleviated if we feed the algorithm with some information about the power in such regions. In that direction, we propose the first algorithm of this work, which is divided into two steps.

(i) Initialization: in this step, we provide the algorithm with information concerning the powers at all directions. However, a high level of accuracy is required to assure a proper behavior of the algorithm. For that reason, we obtain such accuracy by considering the outputs provided by the IAA approach. As reported in [9], the IAA solution is the best beamformer (among the solutions tested there) in terms of power estimation accuracy (numerical evaluation we have performed shows that performance can be seriously degraded if other beamforming solutions are adopted at the first step). The main point here is that the IAA beamformer is not iterated until convergence in this case. Instead, a reduced number of iterations, $N_{\text {it1 } 1}$, are performed (satisfactory results are obtained in our experiments by considering $N_{\text {it1 }}=3$ ).

(ii) IAA Powers and Beamforming Updating: once the initialization is performed, matrix $\hat{\mathbf{P}}$ is constructed by considering the set of powers estimated at the first step. By sorting all these powers in ascending order, $\hat{P}_{r_{1}} \leq \hat{P}_{r_{2}} \leq \cdots \leq \hat{P}_{r L}$, we rewrite matrix $\overline{\mathbf{R}}=\mathbf{A}(\boldsymbol{\theta}, \boldsymbol{\phi}) \widehat{\mathbf{P A}}^{H}(\boldsymbol{\theta}, \boldsymbol{\phi})$ as

$$
\begin{aligned}
\overline{\mathbf{R}}= & \sum_{t=1}^{R-1} \hat{P}_{r t} \mathbf{a}\left(\theta_{r t}, \phi_{r t}\right) \mathbf{a}^{H}\left(\theta_{r t}, \phi_{r t}\right) \\
& +\sum_{t=R}^{L} \hat{P}_{r t} \mathbf{a}\left(\theta_{r t}, \phi_{r t}\right) \mathbf{a}^{H}\left(\theta_{r t}, \phi_{r t}\right) \\
= & \mathbf{Q}+\sum_{t=R}^{L} \hat{P}_{r t} \mathbf{a}\left(\theta_{r t}, \phi_{r_{t}}\right) \mathbf{a}^{H}\left(\theta_{r t}, \phi_{r t}\right),
\end{aligned}
$$

where matrix $\mathbf{Q}$ has been defined as $\mathbf{Q}=$ $\sum_{t=1}^{R-1} \hat{P}_{r_{t}} \mathbf{a}\left(\theta_{r t}, \phi_{r t}\right) \mathbf{a}^{H}\left(\theta_{r t}, \phi_{r t}\right)$ and $R$ is the position of the first power value assuring that

$$
\widehat{P}_{r R}>\beta \widehat{P}_{r 1}
$$

In other words, we divide the total set of powers into two groups, that is, $\left(P_{r_{1}}, \ldots, P_{r_{R-1}}\right)$ and $\left(P_{r_{R}}, \ldots, P_{r_{L}}\right)$. Then, the powers and beamforming vectors are updated, but, however, only the elements belonging to the second group are taken into consideration. In particular, the recursive algorithm presented in Algorithm 3 is carried out. In this step, good results are obtained with $N_{\text {it } 2}=9$ iterations and by setting $\beta$ equal to 2 .

As observed, the second part of the algorithm basically consists of updating only those positions with valuable information. By restricting the search to the directions with initial power $3 \mathrm{~dB}$ higher than the minimum one, we are omitting those directions with only noise contribution. However, the power estimate of these directions contains useful information as it has been estimated at the first step with (a reduced version of) the IAA approach. 


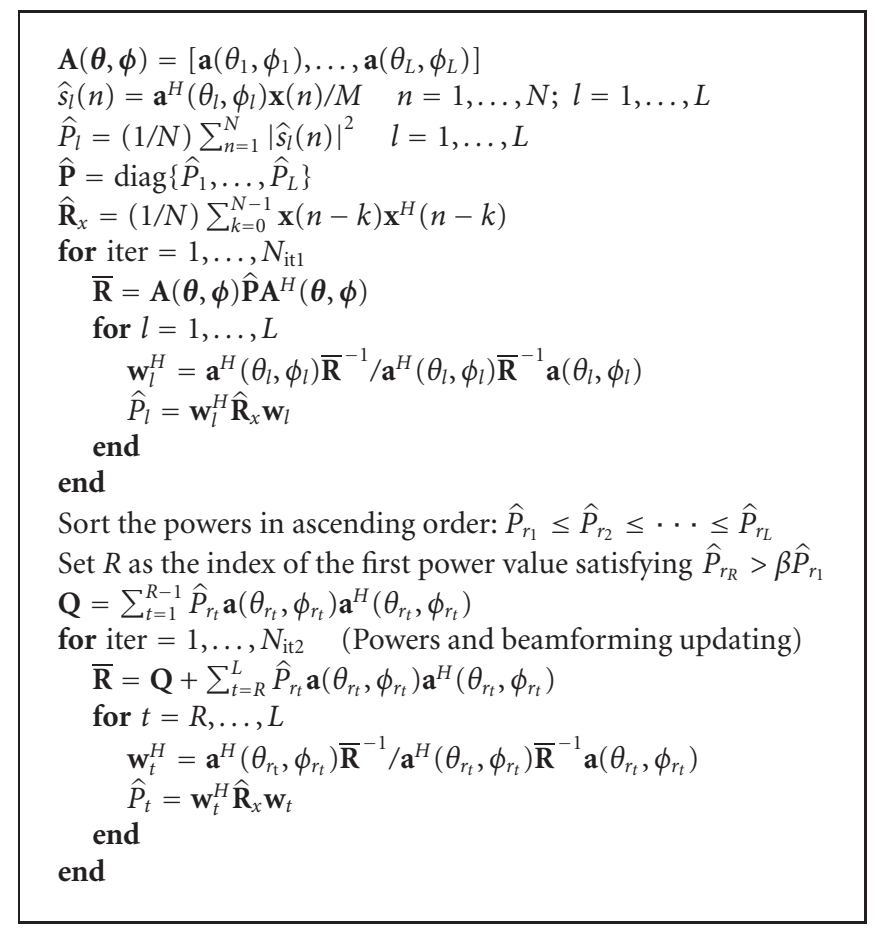

Algorithm 3: IAA-RC algorithm.

Notice that the proposed algorithm is based on the adoption of a power threshold (given by (9)) instead of the direct control of parameter $R$. Clearly, system performance depends on the value of $R$ and an appropriate value trading off complexity versus performance should be selected. However, obtaining the optimal value of $R$ is a critical task as it has a high dependency on the kind of scenario. By adopting a power threshold, we avoid this problem as the algorithm automatically selects those directions providing useful information.

Finally, it is worth mentioning that the proposed solution is totally different from the IAA-Regularized algorithm presented in [11]. In that paper, the objective was to consider directions outside the measurement region of the radar (covering a restricted angular region). To do so, the authors proposed a regularized version of the covariance matrix $\overline{\mathbf{R}}$ consisting of the inclusion of the unknown noise powers omitted by their original scheme. However, these additional noise powers are computed at each iteration of the algorithm, and then computational complexity is increased.

4.2. Solution 2: IAA-ML-RC. The second solution is based on the derivation of a reduced-complexity version of the IAA-ML solution, referred to here as IAA-ML-RC. As in the previous case, the algorithm is divided into two steps.

(i) Initialization: this step is common to the first stage of the IAA-RC approach. Since the IAA beamformer provides the most accurate power estimates, we consider this solution as the initialization phase. It is also worth noting that it is preferable to consider the IAA scheme in this initial phase (instead of the
IAA-ML one), as the algorithm is devoted to find the presence of potential sources with a reduced number of iterations. In summary, the first step of IAA-MLRC consists of conducting $N_{\text {itl }}$ iterations of the IAA approach in the whole visible region.

(ii) IAA-ML Powers Updating: we also consider in this case the powers estimated at the first step, and we rewrite matrix $\overline{\mathbf{R}}$ as (8) by sorting the obtained power estimates and considering the rule given by (9). Then, the IAA-ML algorithm is started by only updating the powers of the group $\left(P_{r_{R}}, \ldots, P_{r_{L}}\right)$ as shown in Algorithm 4. As in the IAA-RC case, satisfactory results can be obtained with IAA-ML-RC by setting the number of iterations equal to $N_{\text {it1 }}=3$ and $N_{\text {it2 }}=$ 9.

4.2.1. Complexity Analysis. Before analyzing the computational complexity of the proposed solutions, we start by studying the complexity of the original IAA and IAA-ML algorithms (where we assume that $L \gg 1$ and $M \gg 1$ ).

(i) IAA Complexity: the number of required operations for the initial stage (Algorithm 1(a) and 1(b)) is $M L+$ $(N+1) L$ products and $M L+(N-1) L$ additions. The computation of the inverse of the matrix given by Algorithm 1(c) (required at the next steps) needs $M^{2} L+M L+21 M^{3}$ products and $M^{2} L+21 M^{3}$ additions. Instead of computing the inverse of this matrix at each iteration, we compute once at the beginning and update it in the algorithm loop in a similar way as done in the case of the IAA-ML algorithm (see Algorithm 2(a)). As a result, the operations required 


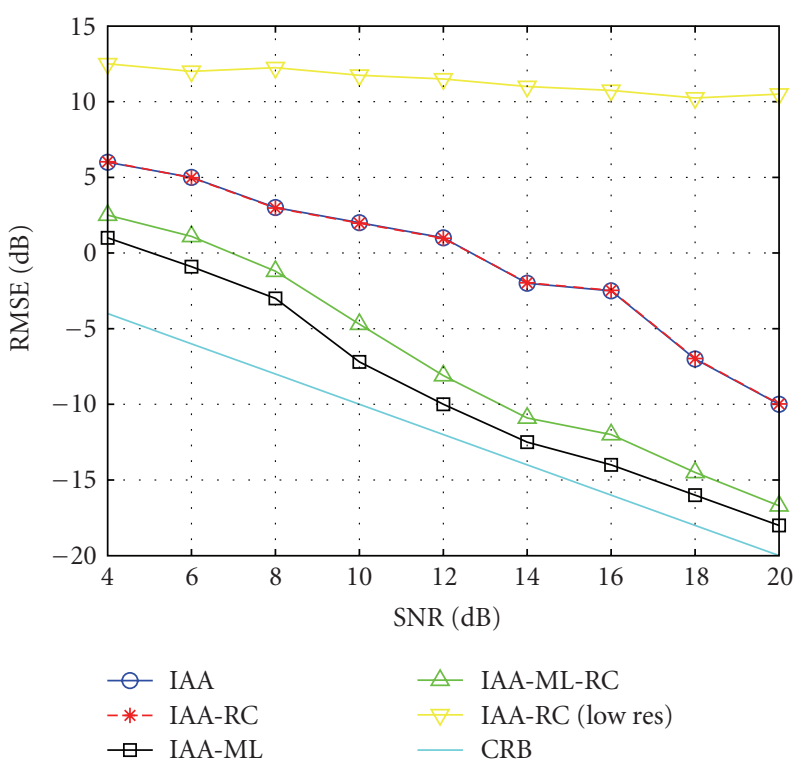

(a)

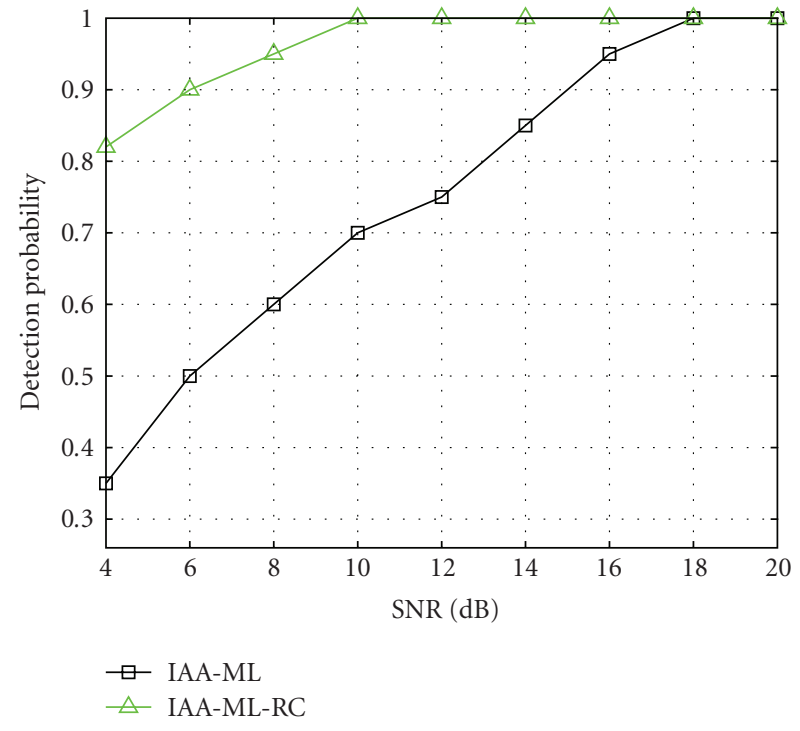

(b)

Figure 11: (a) RMSE of the different beamforming solutions. (b) IAA-ML versus IAA-ML-RC detection probability (rectangular array, $\theta_{1}=10^{\circ}, \phi_{1}=150^{\circ}, \theta_{2}=40^{\circ}, \phi_{2}=188^{\circ}, \theta_{3}=20^{\circ}, \phi_{3}=250^{\circ}, N=10$ snapshots, noise power equal to $0 \mathrm{~dB}$, and $f_{c}=1575 \mathrm{MHz}$ ).

at each iteration of the main core of the algorithm are $7 M^{2} L+3 M L+L$ products and $6 M^{2} L+2 M L+L$ additions are required. By considering $N_{\text {it }}$ iterations, the total complexity amounts to $\left(7 N_{\mathrm{it}}+1\right) M^{2} L+$ $\left(3 N_{\text {it }}+2\right) M L+\left(N_{\text {it }}+N+1\right) L+21 M^{3}$ products and $\left(6 N_{\text {it }}+1\right) M^{2} L+\left(2 N_{\text {it }}+1\right) M L+\left(N_{\text {it }}+N-1\right) L+21 M^{3}$ additions.

(ii) IAA-ML Complexity: the number of operations required for the initial stage and the first matrix inversion are the same as that presented in the IAA case. As for the number of operations needed at each iteration of the main part of the algorithm, this value is equal to $7 M^{2} L+2 M L+3 L$ products and $6 M^{2} L+$ $3 M L+2 L$ additions. As a result, the total complexity is $\left(7 N_{\text {it }}+1\right) M^{2} L+\left(2 N_{\text {it }}+2\right) M L+\left(3 N_{\text {it }}+N+1\right) L+21 M^{3}$ products and $\left(6 N_{\text {it }}+1\right) M^{2} L+\left(3 N_{\text {it }}+1\right) M L+\left(2 N_{\text {it }}+\right.$ $N-1) L+21 M^{3}$ additions.

Concerning the complexity of the proposed IAA-RC and IAA-ML-RC solutions, the following quantities are given.

(iii) IAA-RC Complexity: in the initialization step of the algorithm, the complexity is the number of operations required to conduct the IAA algorithm $N_{\text {itl }}$ iterations, that is $\left(7 N_{\text {itl }}+1\right) M^{2} L+\left(3 N_{\text {it1 }}+\right.$ 2) $M L+\left(N_{\text {it } 1}+N+1\right) L+21 M^{3}$ products and $\left(6 N_{\text {it } 1}+\right.$ 1) $M^{2} L+\left(2 N_{\text {it } 1}+1\right) M L+\left(N_{\text {it } 1}+N-1\right) L+21 M^{3}$ additions. In the second step, $N_{\mathrm{it} 2}$ iterations of the main loop of IAA algorithm must be conducted but only considering $L_{\mathrm{RC}}=L-R+1$ directions. This amounts to $7 N_{\mathrm{it} 2} M^{2} L_{\mathrm{RC}}+3 N_{\mathrm{it} 2} M L_{\mathrm{RC}}+N_{\mathrm{it} 2} L_{\mathrm{RC}}$ products and $6 N_{\mathrm{it} 2} M^{2} L_{\mathrm{RC}}+2 N_{\mathrm{it} 2} M L_{\mathrm{RC}}+N_{\mathrm{it} 2} L_{\mathrm{RC}}$ additions. For the ease of notation, we define $\alpha=$
$(L-R+1) / L$ and express $L_{\mathrm{RC}}$ as $L_{\mathrm{RC}}=\alpha L$. Then, the total number of operations required by IAA-RC can be expressed as $\left(7 N_{\text {it } 1}+7 \alpha N_{\text {it } 2}+1\right) M^{2} L+\left(3 N_{\text {it } 1}+\right.$ $\left.3 \alpha N_{\text {it } 2}+2\right) M L+\left(N_{\text {it } 1}+\alpha N_{\text {it } 2}+N+1\right) L+21 M^{3}$ products and $\left(6 N_{\mathrm{it} 1}+6 \alpha N_{\mathrm{it} 2}+1\right) M^{2} L+\left(2 N_{\mathrm{it} 1}+2 \alpha N_{\mathrm{it} 2}+1\right) M L+$ $\left(N_{\text {it1 }}+\alpha N_{\text {it2 }}+N-1\right) L+21 M^{3}$ additions.

(iv) IAA-ML-RC Complexity: the complexity of the first step of the algorithm is the same as in the previous case. Concerning the power updating phase, the complexity is given by the number of operations of the main loop of the IAA-ML algorithm with $N_{\text {it2 }}$ iterations and $L_{\mathrm{RC}}=\alpha L$ directions: $7 \alpha N_{\mathrm{it} 2} M^{2} L+$ $2 \alpha N_{\mathrm{it} 2} M L+3 \alpha N_{\mathrm{it} 2} L$ products and $6 \alpha N_{\mathrm{it} 2} M^{2} L+$ $3 \alpha N_{\text {it } 2} M L+2 \alpha N_{\text {it } 2} L$ additions. Then, the total number of operations is $\left(7 N_{\text {it } 1}+7 \alpha N_{\text {it } 2}+1\right) M^{2} L+\left(3 N_{\text {it1 }}+\right.$ $\left.2 \alpha N_{\text {it } 2}+2\right) M L+\left(N_{\text {it } 1}+3 \alpha N_{\text {it } 2}+N+1\right) L+21 M^{3}$ products and $\left(6 N_{\text {it } 1}+6 \alpha N_{\text {it } 2}+1\right) M^{2} L+\left(2 N_{\text {it } 1}+3 \alpha N_{\text {it } 2}+1\right) M L+$ $\left(N_{\text {it } 1}+2 \alpha N_{\text {it } 2}+N-1\right) L+21 M^{3}$ additions.

Finally, we present the computational complexity of the different algorithms expressed in terms of floating point operations (FLOPS), where we have considered that one complex product and one complex addition need 6 and 2 FLOPS, respectively. In the scenarios considered in this work (presented and discussed in the next section), the parameters assuring a good performance behavior are $N_{\text {it }}=12, N_{\text {it1 }}=$ 3 , and $N_{\text {it2 }}=9$. The reduction of directions resulting from applying (9) is approximately $\alpha=0.5$ in average. As observed in Table 1, the proposed IAA-RC and IAA-MLRC solutions provide a $37 \%$ of complexity reduction. As we show in the next section, this reduction comes with a residual performance degradation. Besides, we include here the computational complexity of IAA-RC when the rule to 




Algorithm 4: IAA-ML-RC Algorithm.

TABle 1: Computational complexity.

\begin{tabular}{llllll}
\hline & IAA & IAA-ML & IAA-RC & $\begin{array}{l}\text { IAA-RC } \\
(6 \mathrm{~dB})\end{array}$ & $\begin{array}{l}\text { IAA-ML- } \\
\text { RC }\end{array}$ \\
\hline GigaFLOPS & 27.88 & 27.83 & 17.56 & 13.43 & 17.54 \\
\hline
\end{tabular}

update power takes into account a minimum of $\widehat{P}_{r 1}(\mathrm{dBW})+$ $6 \mathrm{~dB}$ (see column labeled as IAA-RC $(6 \mathrm{~dB})$ in the table). In the scenarios considered in this work, we have not observed any performance degradation and complexity can be reduced by $52 \%$ with respect to the IAA case. However, we focus the analysis carried out in the next section on the $\beta=2$ case, as this value assures a good performance behavior for both the IAA-RC and IAA-ML-RC approaches.

\section{Numerical Results}

In this section, we analyze the proposed reducedcomplexity beamformers and compare them with the original IAA approaches in terms of angle estimation capability. In $[9,10]$, the superiority of the IAA approaches with respect to other beamforming solutions is shown in scenarios with array perturbations, finite-sample effects, and coherent and uncorrelated sources. For that reason, we concentrate our efforts here on proving that our solutions are able to provide similar results to those offered by the IAA and IAAML beamformers with a lower computational complexity. Given the recent interest on designing high-performance tracking stations achieving centimeter level tracking accuracy for the Galileo ground mission segment, we consider a scenario with coherent sources as it represents the problem observed in Galileo to separate the line-of-sight signal from the multipath components. Also, we consider some of the parameters of L1 Galileo signal such as the carrier frequency equal to $1575 \mathrm{MHz}$ and the typical signal-to-noise ratio (SNR) levels that can be found at the postcorrelation level (around $10-15 \mathrm{~dB}$ ). As previously mentioned, we adopt a URA with $M=8 \times 8$ antennas.

We start the analysis by showing the performance of the different beamformers in a scenario with three coherent sources at $\left(\theta_{1}=45^{\circ}, \phi_{1}=150^{\circ}\right),\left(\theta_{2}=45^{\circ}, \phi_{2}=188^{\circ}\right)$, and $\left(\theta_{3}=45^{\circ}, \phi_{3}=250^{\circ}\right)$. The received signal-to-noise ratio for these signals is $10 \mathrm{~dB}$. In particular, this value is equal to the signal power as we consider $0 \mathrm{~dB}$ of noise power. We take into account $N=10$ snapshots to compute the beamforming vectors, and the construction of matrix $\mathbf{A}(\boldsymbol{\theta}, \boldsymbol{\phi})$ is carried out with one degree of resolution. Results are shown in Figure 3, where power estimates for the different solutions are represented as a function of the $\phi$ angle (several realizations are plotted in the same figure). Notice that we have considered the same elevation for all the sources for ease of results representation. The circles and vertical lines correspond to the actual sources' powers and angle locations, respectively. As observed the highest resolution is obtained with the IAA-ML-based solutions. However, it is observed that both the IAA and IAA-ML approaches are able to accurately estimate the location of the sources. 




(a)

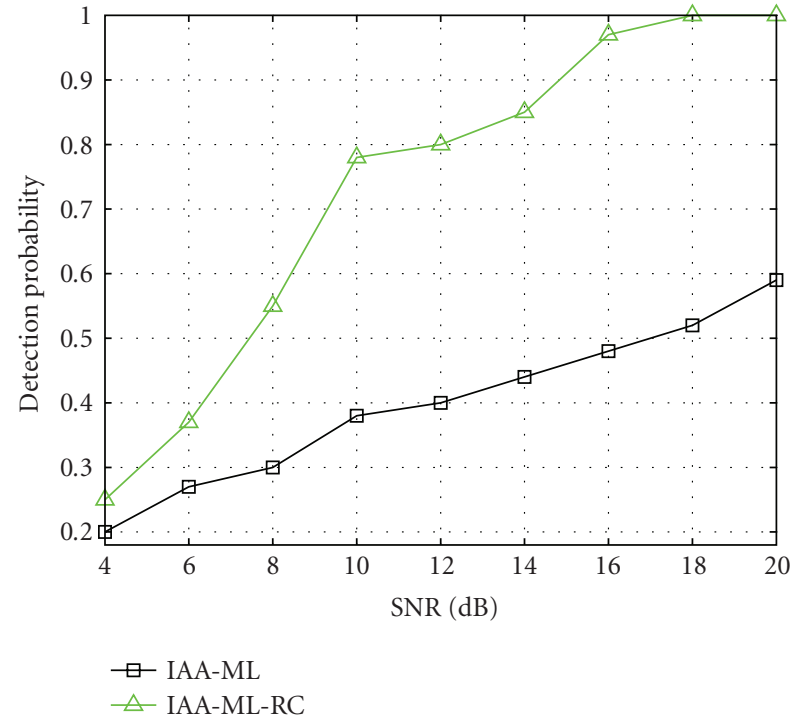

(b)

Figure 12: (a) RMSE of the different beamforming solutions. (b) IAA-ML versus IAA-ML-RC detection probability (rectangular array, $\theta_{1}=10^{\circ}, \phi_{1}=150^{\circ}, \theta_{2}=40^{\circ}, \phi_{2}=188^{\circ}, \theta_{3}=20^{\circ}, \phi_{3}=250^{\circ}, N=3$ snapshots, noise power equal to $0 \mathrm{~dB}$, and $f_{c}=1575 \mathrm{MHz}$ ).

Concerning the IAA-RC and IAA-ML-RC approaches, these provide results quite similar to those attained with IAA and IAA-ML, respectively. Therefore, our solutions are quite efficient to estimate coherent sources' locations with a lower computational complexity. In addition, we also include results corresponding to a low-resolution version of IAA-RC consisting of a first phase with 2 degrees of resolution and a second phase with 1 degree resolution. The motivation of this experiment is to evaluate whether a good performance level can be guaranteed by exploring the area at the first step with a lower resolution. Although complexity can be further reduced, IAA-RC (low res) results present some bias with respect to the other solutions.

In Figure 4, we consider the same scenario, but the number of snapshots is reduced to $N=3$. In this case, it is observed the robustness of IAA against finite-sampling effects as IAA and IAA-RC solutions show a similar behavior to that observed in the $N=10$ case. Concerning the IAAML-based solutions, performance is degraded as one of the problems of IAA-ML is the need of a higher number of snapshots [10]. Indeed, it is observed that situations where some of the sources are not detected by IAA-ML can appear (in the previous case with $N=10$ snapshots, the same problem can appear but with a lower probability). This effect is not observed in this figure as several realizations are plotted. For that reason, we present IAA-ML and IAAML-RC results for only one realization in Figure 5. In that case, IAA-ML is only able to detect two sources. IAA-ML-RC, on the other hand, detects the three sources. Our solution, therefore, provides an improvement of the IAA-ML solution in scenarios with a reduced number of snapshots. This is because the first step of IAA-ML-RC consists of the use of IAA for the initial power estimates, which increases the detection probability of the algorithm.

A different scenario is adopted in Figure 6. In particular, we consider sources' locations at $\left(\theta_{1}=45^{\circ}, \phi_{1}=170^{\circ}\right)$, $\left(\theta_{2}=45^{\circ}, \phi_{2}=188^{\circ}\right)$, and $\left(\theta_{3}=45^{\circ}, \phi_{3}=250^{\circ}\right)$, and the number of snapshots is equal to $N=10$. Again, the reducedcomplexity versions provide similar results to those attained with the original algorithms. Nevertheless, in this case where the first and second sources are closer, performance of the different algorithms are degraded with respect to the previous scenario. The IAA-ML-based solutions provide the best results due to their higher resolution. It is worth recalling that several realizations are plotted and for that reason several peaks are observed around the actual locations (one peak for realization). In the case of the IAA and IAA-RC approaches, the algorithms are not able to properly differentiate the two first sources. Therefore, this kind of environment is clearly the most appropriate scenario to use IAA-ML solutions. By reducing the number of snapshots to $N=3$ (see Figure 7), the performance of the different algorithms remain the same except for the IAA-ML solutions. In other words, IAA-ML shows performance degradation at the presence of finite sampling effects. As commented in the cases represented by Figures 4 and 5, the IAA-ML-RC approach presents a more robust option when the number of snapshots is reduced. Then, this solution is an appropriate choice to cope with sources at close locations in scenarios with a low number of snapshots. As computational complexity is also reduced, this solution can also be efficient in situations with a high number of snapshots. Notice that IAA-RC (low-res) is able to separate the two sources but a higher bias is presented and more sources than the actual number can be detected in some situations (see Figure 8). 
In Figure 9, we represent the root mean square error (RMSE) in terms of bearing accuracy (i.e., error in DOA estimation) attained with the different beamforming solutions in the same scenario adopted by Figure 3 but by considering different values of SNR. The Cramer-Rao Bound (CRB) is also included for benchmarking [12, Chapter 8]. As observed, the highest resolution is obtained with the IAA-ML based solutions. On the other hand, one can see how results of the proposed reduced complexity solutions are quite close to those achieved by the original schemes, especially in the IAA-RC case. Concerning the IAA-RC (lowres) approach, it is emphasized the bad behavior offered by reducing the resolution at the first step of the algorithm. It is worth noting that we have generated these curves by neglecting the nondetection probability of the IAA-ML schemes commented above (i.e., RMSE is only accounted for good detection events). In order to show this last effect, we present in the right-hand side of the figure the detection probability for both approaches. By considering the signals with the three largest powers, the detection probability for a given source is defined as the probability that a signal is present inside the area surrounding the exact location of that source (where the area is defined as the region at $\pm 5^{\circ}$ of the exact DOA of the source). As observed, a higher detection probability is observed for the IAA-ML-RC case when compared with the IAA-ML solution. As previously commented, this is because IAA-ML-RC adopts IAA for the initial power estimates. In Figure 10, we consider the same scenario, but the number of snapshots is reduced to $N=3$. Here, it is observed how performance of IAA-MLbased schemes are degraded with respect to the previous case. In particular, performance of IAA-ML-RC is better than the IAA-ML one in this case. Concerning the detection probability, the higher robustness against finite-sample effect of the IAA-ML-RC is emphasized here.

As commented above, results have been presented in scenarios with sources at the same elevation for ease of results representation. Although this is still a $2 \mathrm{D}$ DOA estimation problem as the search procedure carried out by the different algorithms is performed for the whole $(\theta, \phi)$ space, we include here results corresponding to a scenario with the following sources' locations $\left(\theta_{1}=10^{\circ}, \phi_{1}=150^{\circ}\right),\left(\theta_{2}=\right.$ $\left.40^{\circ}, \phi_{2}=188^{\circ}\right)$, and $\left(\theta_{3}=20^{\circ}, \phi_{3}=250^{\circ}\right)$. More specifically, results in terms of RMSE curves are presented in Figures 11 and 12 for the cases of $N=10$ and 3 snapshots, respectively. As observed, similar conclusions to those extracted in the previous scenario can be obtained here.

In summary, the following conclusions can be drawn from observed results:

(i) IAA-RC provides similar results to those offered by the original IAA algorithm, while computational complexity can be significantly reduced,

(ii) IAA-ML-RC suffers from a minor performance degradation with respect to IAA-ML, but substantial computational savings can also be obtained. When the system is restricted to work with a reduced number of snapshots, however, IAA-ML-RC is able to provide a more robust solution than IAA-ML. This is because the first step of the algorithm is based on IAA,

(iii) IAA-ML-RC offers higher-resolution levels than that offered by IAA-RC (with a sufficient number of snapshots),

(iv) IAA-RC could be a more convenient solution when the system is complexity limited and attaining highresolution levels is not the main motivation. It is also an appropriate solution to cope with finite-sample effects.

\section{Conclusions}

In this paper, we have considered the problem of $2 \mathrm{D}$ angle estimation in scenarios with coherent sources. To do so, we have adopted beamforming designs based on the IAA and IAA-ML solutions. Since the computational complexity of these schemes can be prohibitive in real applications, we have proposed two beamformers with reduced complexity. On one hand, we have proposed a reduced complexity version of IAA referred to here as IAA-RC, which offers similar results to the original algorithm while reducing complexity by a $52 \%$. It has been shown that this algorithm is quite appropriate for dealing with scenarios with a few of snapshots and lower requirements in terms of angle estimation resolution. On the other hand, a reduced version of IAA-ML, namely IAA-ML-RC, has also been derived. In this case, we have shown that high-resolution results can be attained by reducing the complexity of IAA-ML by $37 \%$. Besides, IAA-ML-RC is also able to alleviate the finitesample effects of IAA-ML as the initial step of the proposed algorithm is based on the IAA approach.

\section{Acknowledgments}

This work was supported by the Spanish Government Project TEC2008-06305/TEC, the Catalan Government under Grant 2009 SGR 298, and the Chair of Knowledge and Technology Transfer Parc de Recerca UAB-Santander.

\section{References}

[1] F. Amarillo, A. Ballereau, M. Crisci, B. Lobert, and S. Lannelongue, "Galileo IOV ground mission segment performances," in Proceedings of European Navigation Conference (ENC-GNSS '08), April 2008.

[2] G. Seco-Granados, J. A. Fernández-Rubio, and C. FernándezPrades, "ML estimator and hybrid beamformer for multipath and interference mitigation in GNSS receivers," IEEE Transactions on Signal Processing, vol. 53, no. 3, pp. 1194-1208, 2005.

[3] J. Lopez Vicario, F. Antreich, M. Barcelo et al., "ADIBEAM: adaptive digital beamforming for Galileo reference ground stations," in Proceedings of the Institute of Navigation (IONGNSS '10), September 2010.

[4] R. O. Schmidt, "Multiple emitter location and signal parameter estimation," IEEE Transactions on Antennas and Propagation, vol. 34, no. 3, pp. 276-280, 1986.

[5] M. Haardt and J. A. Nossek, "Unitary ESPRIT: how to obtain increased estimation accuracy with a reduced computational 
burden," IEEE Transactions on Signal Processing, vol. 43, no. 5, pp. 1232-1242, 1995.

[6] T. J. Shan and T. Kailath, "Adaptive beamforming for coherent signals and interference," IEEE Transactions on Acoustics, Speech, and Signal Processing, vol. 33, no. 3, pp. 527-536, 1985.

[7] H. Krim and M. Viberg, "Two decades of array signal processing research: the parametric approach," IEEE Signal Processing Magazine, vol. 13, no. 4, pp. 67-94, 1996.

[8] K. S. M. Wax and A. Leshem, "Joint estimation of time delays and directions of arrival of multiple reflections of a," IEEE Transactions on Signal Processing, vol. 45, no. 10, pp. 24772484, 1997.

[9] L. Du, T. Yardibi, J. Li, and P. Stoica, "Review of user parameter-free robust adaptive beamforming algorithms," Digital Signal Processing, vol. 19, no. 4, pp. 567-582, 2009.

[10] T. Yardibi, J. Li, P. Stoica, M. Xue, and A. B. Baggeroer, "Source localization and sensing: a nonparametric iterative adaptive approach based on weighted least squares," IEEE Transactions on Aerospace and Electronic Systems, vol. 46, no. 1, pp. 425-443, 2010.

[11] W. Roberts, P. Stoica, J. Li, T. Yardibi, and F. A. Sadjadi, "Iterative adaptive approaches to MIMO radar imaging," IEEE Journal on Selected Topics in Signal Processing, vol. 4, no. 1, pp. 5-20, 2010.

[12] H. V. Trees, Optimum Array Processing (Detection, Estimation and Modulation Theory, Part IV), John Wiley \& Sons, New York, NY, USA, 2002. 